\title{
Qualities of the axion and LSP in Pati-Salam unification with $\mathbb{Z}_{4}^{R} \times \mathbb{Z}_{N}$ symmetry
}

\author{
Junichiro Kawamura $\oplus^{1,2, *}$ and Stuart Raby $\circledast^{1, \dagger}$ \\ ${ }^{1}$ Department of Physics, Ohio State University, Columbus, Ohio 43210, USA \\ ${ }^{2}$ Department of Physics, Keio University, Yokohama 223-8522, Japan
}

(Received 16 September 2020; accepted 9 December 2020; published 4 January 2021)

\begin{abstract}
In this paper we construct supersymmetric Pati-Salam (PS) models containing the minimal supersymmetric standard model and an invisible axion. The models include two discrete symmetries, $\mathbb{Z}_{4}^{R} \times \mathbb{Z}_{N}$, which maintain the quality of the accidental Peccei-Quinn (PQ) symmetry and thus the solution to the strong $C P$ problem. We require that the discrete anomaly conditions are satisfied for both $\mathbb{Z}_{4}^{R} \times G_{\mathrm{PS}}^{2}$ and $\mathbb{Z}_{N} \times G_{\mathrm{PS}}^{2}$. The vacuum expectation value of the $\mathrm{PQ}$ field spontaneously breaks all the discrete symmetries. $\mathrm{R}$-parity is violated if any of the PQ field(s) has an odd charge under $\mathbb{Z}_{4}^{R}$. We present two explicit models which we refer to as a minimal model where R-parity violation is extremely suppressed, and a nonminimal model where R-parity violation is significant. In the latter model, the neutralino becomes unstable even if it is the lightest supersymmetric particle (LSP), and, in addition, there are new low-energy vectorlike states. In both examples, R-parity violation is sufficiently suppressed such that the proton is stable.
\end{abstract}

DOI: $10.1103 /$ PhysRevD.103.015002

\section{INTRODUCTION}

The Peccei-Quinn (PQ) symmetry, $U(1)_{\mathrm{PQ}}$, provides an attractive solution to the strong $C P$ problem $[1,2]$. The $\theta$ angle in quantum chromodynamics (QCD) settles at zero dynamically due to the potential of a pseudo NambuGoldstone boson of PQ symmetry breaking, i.e., the socalled axion, generated through QCD quantum effects $[3,4]$. Since the PQ symmetry is an anomalous global symmetry, it will be broken by quantum gravity effects. However, the PQ breaking effects should be extremely suppressed such that the QCD axion potential still has a minimum at $|\theta|<10^{-10}$ to be consistent with the measurements of the neutron electric dipole moment [5-7]. This problem is known as the axion quality problem [8-12].

In this paper, we propose simple models with Pati-Salam (PS) gauge symmetry and non-anomalous discrete symmetries, $\mathbb{Z}_{4}^{R} \times \mathbb{Z}_{N}$, where $N$ is an integer. We aim to construct models in which the PQ symmetry arises as an accidental symmetry and its quality is ensured by the discrete symmetries. ${ }^{1}$ The Pati-Salam (PS) unification [22] of the Standard Model (SM) is attractive because the SM

\footnotetext{
*kawamura.14@osu.edu

†raby.1@osu.edu

${ }^{1}$ See Refs. [13-21] for solutions to the quality problem.
}

Published by the American Physical Society under the terms of the Creative Commons Attribution 4.0 International license. Further distribution of this work must maintain attribution to the author(s) and the published article's title, journal citation, and DOI. Funded by SCOAP ${ }^{3}$. fermions are unified into two multiplets, hypercharge is quantized, and the proton is not destabilized by exotic gauge/Higgs bosons. Although the PS gauge symmetry, $G_{\mathrm{PS}}:=S U(4)_{C} \times S U(2)_{L} \times S U(2)_{R}$, is not grand unified to a simple group, the PS model can be realized in an orbifold grand unification theory (GUT) in extra dimensions and from the heterotic string, for example, see Ref. [23,24]. Thus gauge coupling unification can be assumed with small threshold corrections at the GUT/ compactification scale. It has been shown that the recent experimental data can be explained very precisely in the PS model $[25,26]$.

We will consider PS models with supersymmetry (SUSY) and the discrete R-symmetry. The minimal supersymmetric Standard Model (MSSM) is an attractive candidate for a model at the $\mathrm{TeV}$ scale, since it solves the gauge hierarchy problem, three gauge couplings constants are unified at the GUT scale and the electroweak (EW) symmetry breaking is triggered radiatively. The $\mathbb{Z}_{4}^{R}$ symmetry is a unique anomaly-free symmetry consistent with the PS unification which can forbid the dimension-4 and dimension-5 operators responsible for proton decay, as well as the mass term of the Higgs doublets at the Planck scale $[27,28]$. Without the PQ field, R-parity exists exactly if the $\mathbb{Z}_{4}^{R}$ symmetry is broken by nonperturbative effects associated with SUSY breaking, and thus the lightest supersymmetric particle (LSP) will contribute to the dark matter (DM).

We also introduce a nonanomalous $\mathbb{Z}_{N}$ symmetry to solve the axion quality problem. Since the PQ field carries charges under both $\mathbb{Z}_{4}^{R}$ and $\mathbb{Z}_{N}$ symmetries, the nonzero 
vacuum expectation value (VEV) of it will break the discrete symmetries. Hence, there can be R-parity violation (RPV) due to the spontaneous breaking of the $\mathbb{Z}_{4}^{R}$ symmetry, if the PQ field carries odd R-charge. In this case we consider two viable scenarios of the unstable LSP. One is that the RPV effect is so suppressed that the lifetime of the LSP is much longer than the age of the universe. In this case, the low-energy R-parity is accidental, but is high quality such that the LSP contributes to the DM. The other scenario is that the RPV is so large that the LSP decays before big bang nucleosynthesis (BBN), and thus the LSP is not the DM, i.e., the R-parity is low quality. The intermediate case is excluded by experiments [29-33]. We will show examples for each of these scenarios.

The rest of this paper is organized as follows. We introduce our generic model in Sec. II. Two models satisfying all the constraints are discussed in Sec. III. We conclude this paper in Sec. IV. The Higgs potential in our nonminimal model with an extra bidoublet field is discussed in Appendix A. The sizes of coupling constants of operators in our examples are listed in Appendix B.

\section{GENERIC MODEL}

We introduce SUSY models based on the Pati-Salam gauge symmetry, which is broken down to the SM at the GUT scale, and the discrete $\mathbb{Z}_{4}^{R}$ and $\mathbb{Z}_{N}$ symmetries. The goal of the present paper is to study the conditions under which

(1) the mixed anomalies of the discrete/PS symmetries $\mathbb{Z}_{4}^{R} \times G_{\mathrm{PS}}^{2}$ and $\mathbb{Z}_{N} \times G_{\mathrm{PS}}^{2}$ cancel.

(2) the anomalous $U(1)_{\mathrm{PQ}}$ symmetry is realized accidentally and is so high quality that it solves the strong $C P$ problem.

(3) the $\mu / b$-term are generated around the SUSY breaking scale.

(4) any particles not in the MSSM are sufficiently heavy and quickly decaying.

(5) the gauge coupling constants are unified at a high scale.

The conditions (1) and (2) are for the nonanomalous discrete symmetry explanation for the strong $C P$ problem. The conditions (3) and (4) are phenomenological requirements. The condition (5) may not be necessary for the Pati-Salam unification, but the gauge coupling unification allows us to interpret this model as the 4 dimensional theory resulting from an orbifold GUT in higher dimensions $[23,24]$. In this paper, the three gauge couplings are assumed to be approximately equal up to threshold corrections at the GUT scale.

The R-parity may also arise accidentally, and thus the LSP may decay through interactions induced by higherdimensional operators. Phenomenologically viable scenarios are which

(a) the lifetime of the LSP is much longer than the age of universe,

(b) the LSP decays before BBN.
We will show an example for each case in Sec. III.

We will consider the following superpotential,

$$
W=W_{\mathrm{PS}}+W_{\mathrm{PQ}}+\Delta W
$$

Here, $W_{\mathrm{PS}}$ is the leading superpotential including the MSSM fields and fields responsible for the PS breaking. $W_{\mathrm{PQ}}$ is the leading superpotential for the spontaneous PQ breaking sector. $\Delta W$ includes higher-dimensional operators which will induce the $\mu / b$-term for the Higgs doublets as well as explicit PQ breaking and/or RPV. $W_{\mathrm{PS}}$ and $W_{\mathrm{PQ}}$ will be introduced in Secs. II A and II B, respectively. The explicit PQ breaking and RPV are respectively discussed in Secs. II D and II E.

\section{A. Pati-Salam sector}

The matter content of the generic Pati-Salam model is shown in Table I. $\Lambda$ is a cutoff scale for the model. The leading superpotential is given schematically by

$$
\begin{aligned}
W_{\mathrm{PS}}= & Q \mathcal{H} Q^{c}+\frac{1}{2 \Lambda} \bar{S}^{c} Q^{c} \bar{S}^{c} Q^{c} \\
& +X\left(\bar{S}^{c} S^{c}+\bar{\Sigma} \Sigma+\overline{\mathcal{H}} \mathcal{H}-v_{\mathrm{PS}}^{2}\right)+X^{3}+S^{c} \Sigma S^{c} \\
& +\bar{S}^{c} \bar{\Sigma} \bar{S}^{c}+W_{s=0},
\end{aligned}
$$

where

$$
W_{s=0}=\bar{S}^{c} \Sigma \bar{S}^{c}+S^{c} \bar{\Sigma} S^{c},
$$

is allowed only if $4 s \equiv 0$ modulo $N$. The superpotential has R-charge 2 under $\mathbb{Z}_{4}^{R}$, while it is neutral under $\mathbb{Z}_{N}$. Throughout this paper, we omit coupling constants which may be $\mathcal{O}(1){ }^{2}$ The MSSM quarks and leptons are contained in $Q$ and $Q^{c}$ as

$$
Q=\left(\begin{array}{ll}
q & \ell
\end{array}\right), \quad Q^{c}=\left(\begin{array}{ll}
u^{c} & \nu^{c} \\
d^{c} & e^{c}
\end{array}\right),
$$

where the rows are $S U(2)_{R}$ space and columns are the $S U(4)_{C}$ space. Here, the flavor indices are implicit. In the minimal model without $\overline{\mathcal{H}}$, the MSSM Higgs doublets are in the bidoublet $\mathcal{H}$. There are four Higgs doublets in the nonminimal model, and two linear combinations of them correspond to the MSSM-like Higgs doublets, see Appendix A for more details. $S^{c}, \bar{S}^{c}$ are the PS breaking fields whose VEVs are given by $\left\langle S^{c}\right\rangle=v_{\mathrm{PS}} \delta^{4 \alpha} \delta^{i 1}$ and $\left\langle\bar{S}^{c}\right\rangle=v_{\mathrm{PS}} \delta_{4 \alpha} \delta_{i 1}$, where $\alpha$ is the $S U(4)_{C}$ index and $i$ is the $S U(2)_{R}$ index. The Majorana masses of the right-handed neutrinos are generated from the last term on the first line in

\footnotetext{
${ }^{2}$ The hierarchy in the SM Yukawa couplings may be explained by the Froggatt-Nielsen mechanism [34], as studied in Refs. [26,35].
} 
TABLE I. Matter content of the generic model. There are $N_{g}=3$ generations of $Q$ and $Q^{c}$. The fields $\bar{\Sigma}$ and $\overline{\mathcal{H}}$ are not included in our minimal model.

\begin{tabular}{lccccccccc}
\hline \hline & $\mathcal{H}$ & $Q$ & $Q^{c}$ & $X$ & $S^{c}$ & $\bar{S}^{c}$ & $\Sigma$ & $\bar{\Sigma}$ & $\overline{\mathcal{H}}$ \\
\hline$S U(4)_{C}$ & $\mathbf{1}$ & $\mathbf{4}$ & $\overline{\mathbf{4}}$ & $\mathbf{1}$ & $\overline{\mathbf{4}}$ & $\mathbf{4}$ & $\mathbf{6}$ & $\mathbf{6}$ & $\mathbf{1}$ \\
$S U(2)_{L}$ & $\mathbf{2}$ & $\mathbf{2}$ & $\mathbf{1}$ & $\mathbf{1}$ & $\mathbf{1}$ & $\mathbf{1}$ & $\mathbf{1}$ & $\mathbf{1}$ & $\mathbf{2}$ \\
$S U(2)_{R}$ & $\mathbf{2}$ & $\mathbf{1}$ & $\overline{\mathbf{2}}$ & $\mathbf{1}$ & $\overline{\mathbf{2}}$ & $\mathbf{2}$ & $\mathbf{1}$ & $\mathbf{1}$ & $\mathbf{2}$ \\
$\mathbb{Z}_{4 R}$ & 0 & 1 & 1 & 2 & 0 & 0 & 2 & 2 & 0 \\
$\mathbb{Z}_{N}$ & $h$ & $-h-s$ & $s$ & 0 & $s$ & $-s$ & $-2 s$ & $2 s$ & $-h$ \\
\hline \hline
\end{tabular}

Eq. (2) after PS breaking. ${ }^{3}$ The right-handed neutrino mass, $M_{R}$, is then given by

$M_{R} \sim \frac{v_{\mathrm{PS}}^{2}}{\Lambda}=10^{14} \mathrm{GeV} \times\left(\frac{v_{\mathrm{PS}}}{10^{16} \mathrm{GeV}}\right)^{2}\left(\frac{10^{18} \mathrm{GeV}}{\Lambda}\right)$.

A singlet $X$ with R-charge 2 is necessary to break the PS symmetry by the F-term potential. The vacuum which breaks PS down to the SM gauge symmetry, with $\left\langle\bar{S}^{c} S^{c}\right\rangle \neq 0$ and $\langle\bar{\Sigma} \Sigma\rangle=\langle\overline{\mathcal{H}} \mathcal{H}\rangle=\left\langle X^{2}\right\rangle=0$, is a global minimum of the scalar potential in global SUSY. The other directions would be stabilized by e.g., Planck suppressed operators in the Kähler potential and/or SUSY breaking mass terms. The sextet $\Sigma$ forms a mass term with the color antitriplet in $S^{c}$ (and triplet in $\bar{S}^{c}$ if $4 s \equiv 0$ ). The charges under $\mathbb{Z}_{N}$ are chosen to be consistent with the superpotential Eq. (2). ${ }^{4}$

$\bar{\Sigma}$ and $\overline{\mathcal{H}}$ are not included in the minimal model, but are necessary to have sizable RPV interactions consistent with the conditions (1)-(5) as will be discussed in Sec. III B. Without those fields, $4 s \equiv 0$ modulo $N$ is required to make the triplets in both $S^{c}$ and $\bar{S}^{c}$ having masses of $\mathcal{O}\left(v_{\mathrm{PS}}\right)$ via $W_{s=0}$. In the model with $4 s \not \equiv 0$, one of the two color triplets in $\Sigma$ and $\bar{\Sigma}$ are remain massless after the PS breaking. The light (anti-)triplet $\sigma(\bar{\sigma})$ are defined as

$$
\bar{\sigma}^{a}:=\varepsilon^{a b c} \Sigma_{b c}, \quad \sigma_{a}:=\varepsilon_{a b c} \bar{\Sigma}^{b c},
$$

where $a, b, c=1,2,3$ are the color indices. The (anti-)triplets $(\bar{\sigma}, \sigma)$ have hypercharge $(1 / 3,-1 / 3)$, and thus form a vectorlike pair. In this paper, the hypercharge is defined as $Y=(B-L) / 2+T_{3 R}$, where $T_{3 R}$ is a generator of $S U(2)_{R}$ whose eigenvalue is 0 for a singlet, $\pm 1 / 2$ for a doublet. In the nonminimal model, the bidoublets $\mathcal{H}, \overline{\mathcal{H}}$ and the triplets will have mass via

\footnotetext{
${ }^{3}$ This term can be obtained by integrating out a gauge singlet field $N$, which carries charges $(1,0)$ under $\left(\mathbb{Z}_{4}^{R}, \mathbb{Z}_{N}\right)$, from a renormalizable superpotential $\bar{S}^{c} Q N+\frac{1}{2} M_{N} N N$. The mass parameter $M_{N}$ is expected to be $\mathcal{O}(\Lambda)$.

${ }^{4}$ It has been shown that this superpotential is consistent with SUSY hybrid inflation $[35,36]$. The PS breaking fields $S^{c}$ and $\bar{S}^{c}$ play a role of the waterfall fields, so that the PS symmetry is broken during the inflation. Hence, the PS monopole is diluted away.
}

$$
\Delta W \supset w_{0}(\bar{\Sigma} \Sigma+\overline{\mathcal{H}} \mathcal{H})
$$

where $w_{0}$ has charge $(2,0)$ and its size is expected to be the SUSY breaking scale. In general, the VEV of the superpotential in a hidden sector would be a source for $w_{0}$ [37]..$^{5}$ It is remarkable that the vectorlike triplets $(\sigma, \bar{\sigma})$ and doublets in $\overline{\mathcal{H}}$ can be embedded into a vectorlike pair of $(\mathbf{5}, \overline{\mathbf{5}})$ under $S U(5)$. Hence, gauge coupling unification may still hold even with the exotic triplets, if they do not have any other mass larger than $\mathcal{O}\left(w_{0}\right)$ which could, in principle, originate from PQ breaking.

\section{B. Peccei-Quinn sector}

We introduce the PQ fields, $P$ and $\bar{P}$, which carry $\mathbb{Z}_{4}^{R}$ and $\mathbb{Z}_{N}$ charge $(r, p)$ and $(\bar{r}, \bar{p})$, respectively, and the vectorlike PS quarks in Table II. In the minimal model, $\bar{P}$ is not necessary, but is required for sizable RPV interactions in addition to $\bar{\Sigma}$ and $\overline{\mathcal{H}}$. We will consider the Kim-ShifmanVainshtein-Zakharov (KSVZ) axion model with the vectorlike fields $[38,39]$. The superpotential is given by

$$
W_{\mathrm{PQ}}=P \bar{\Psi} \Psi+\bar{P} \bar{\Psi}^{c} \Psi^{c}+W_{\mathrm{dec}},
$$

where $W_{\text {dec }}$ contains interactions for decays of the vectorlike fields. In the minimal model without $\bar{P}$, the second term is replaced by $P \bar{\Psi}^{c} \Psi^{c}$ and the charge of $\bar{\Psi}^{c}$ is given by $\left(2-r-\bar{r}_{\Psi},-p-\bar{p}_{\Psi}\right)$. Since we assume that the vectorlike fields have the same gauge quantum number under the PS symmetry, $W_{\text {dec }}$ will have Yukawa interactions similar to $Q \mathcal{H} Q^{c}$, depending on the charges. In order to preserve gauge coupling unification, we introduce $N_{\Psi}$ pairs of ( $\Psi$, $\bar{\Psi})$ and $\left(\Psi^{c}, \bar{\Psi}^{c}\right)$.

In this paper, we consider that the VEV of $P$ generated by the radiatively corrected soft SUSY breaking mass term, $m_{P}^{2}|P|^{2}[40]^{6}$ The soft SUSY breaking mass squared will be driven to negative values by renormalization group running due to the Yukawa coupling in Eq. (8), so that the nonzero VEV of $P$ is generated by dimensional transmutation [41]. We expect the following form of the scalar potential,

$$
V_{P}=m_{P}^{2}|P|^{2}\left(\log \frac{|P|^{2}}{f_{\mathrm{PQ}}^{2}}-1\right) .
$$

The minimum of this potential is at $f_{\mathrm{PQ}}$ whose scale can be within the so-called axion window, $10^{9}-10^{12} \mathrm{GeV}$, where the QCD axion can be the DM. After PQ breaking, $\mathbb{Z}_{4}^{R}$ symmetry (and simultaneously R-parity) is completely broken if $r= \pm 1$, while R-parity remains unbroken if

\footnotetext{
${ }^{5}$ The mass term $w_{0}$ could also originate from $\langle X\rangle$.

${ }^{6} \mathrm{We}$ could also consider PQ breaking by the tree-level superpotential, $X\left(P \bar{P}-f_{\mathrm{PQ}}^{2}\right)$. However, this may cause a fine-tuning problem, i.e., $f_{\mathrm{PQ}} \ll v_{\mathrm{PS}}$, since we have already introduced the term $X v_{\mathrm{PS}}^{2}$ for PS breaking.
} 
TABLE II. Charges of the PQ fields $P, \bar{P}$ and vectorlike fields. The field $\bar{P}$ is not included in our minimal model.

\begin{tabular}{lcccccc}
\hline \hline & $\bar{\Psi}$ & $\Psi$ & $\Psi^{c}$ & $\bar{\Psi}^{c}$ & $P$ & $\bar{P}$ \\
\hline$S U(4)_{C}$ & $\overline{\mathbf{4}}$ & $\mathbf{4}$ & $\overline{\mathbf{4}}$ & $\mathbf{4}$ & $\mathbf{1}$ & $\mathbf{1}$ \\
$S U(2)_{L}$ & $\overline{\mathbf{2}}$ & $\mathbf{2}$ & $\mathbf{1}$ & $\mathbf{1}$ & $\mathbf{1}$ & $\mathbf{1}$ \\
$S U(2)_{R}$ & $\mathbf{1}$ & $\mathbf{1}$ & $\overline{\mathbf{2}}$ & $\mathbf{2}$ & $\mathbf{1}$ & $\mathbf{1}$ \\
$\mathbb{Z}_{4 R}$ & $2-r-r_{\Psi}$ & $r_{\Psi}$ & $\bar{r}_{\Psi}$ & $2-\bar{r}-\bar{r}_{\Psi}$ & $r$ & $\bar{r}$ \\
$\mathbb{Z}_{N}$ & $-p-p_{\Psi}$ & $p_{\Psi}$ & $\bar{p}_{\Psi}$ & $-\bar{p}-\bar{p}_{\Psi}$ & $p$ & $\bar{p}$ \\
\hline \hline
\end{tabular}

$r=2$. The $\mathbb{Z}_{N}$ symmetry is broken at the PS scale, $v_{\mathrm{PS}}$, if $s \neq 0$, while it is broken at the PQ breaking scale, $f_{\mathrm{PQ}}$, if $s=0$. The same discussion can be applied for the other PQ field $\bar{P}$. In this paper, we assume that the VEVs of the PQ fields are the same scale, i.e., $\langle P\rangle \sim\langle\bar{P}\rangle \sim f_{\mathrm{PQ}}$.

In the nonminimal model, there should be $\mu_{\mathcal{H}} \mathcal{H}^{2}$ so that $\tan \beta \neq \infty$, although the Higgsino masses are explained by $w_{0} \mathcal{H} \overline{\mathcal{H}}$, see Appendix A for more details of the Higgs sector with the extra bidoublet $\overline{\mathcal{H}}$. This term can be explained by the Kim-Nilles mechanism [42] if there is a term

$$
\Delta W \supset \frac{1}{\Lambda}(P, \bar{P})^{2} \mathcal{H}^{2},
$$

where $(P, \bar{P})^{2}=\left\{P^{2}, P \bar{P}, \bar{P}^{2}\right\}$. This term induces the effective $\mu$-term for $\mathcal{H}^{2}$,

$\mu_{\mathcal{H}} \sim \frac{f_{\mathrm{PQ}}^{2}}{\Lambda} \sim 100 \mathrm{GeV} \times\left(\frac{f_{\mathrm{PQ}}}{10^{10} \mathrm{GeV}}\right)^{2}\left(\frac{10^{18} \mathrm{GeV}}{\Lambda}\right)$.

The mass terms ( $b$-terms) for the non-SM Higgs bosons are generated by the SUSY breaking.

\section{Anomaly cancellation}

We denote coefficients of the mixed anomaly of $\mathbb{Z}_{4}^{R}$ to $S U(4)_{C}^{2}, S U(2)_{L}^{2}$ and $S U(2)_{R}^{2}$ by $\mathcal{A}_{C}^{4 R}, \mathcal{A}_{L}^{4 R}$ and $\mathcal{A}_{R}^{4 R}$, respectively. Those of $\mathbb{Z}_{N}$ are denoted by $\mathcal{A}_{C}^{N}, \mathcal{A}_{L}^{N}$ and $\mathcal{A}_{R}^{N}$. The coefficients are given by [43-48],

$\mathcal{A}_{C}^{4 R} \equiv 1+N_{\bar{\Sigma}}-(r+\bar{r}) N_{\Psi}, \quad \mathcal{A}_{L}^{4 R} \equiv 1-N_{\overline{\mathcal{H}}}-2 r N_{\Psi}$,

$\mathcal{A}_{R}^{4 R} \equiv 1-N_{\overline{\mathcal{H}}}-2 \bar{r} N_{\Psi}$,

modulo 2 and

$$
\begin{aligned}
& \mathcal{A}_{C}^{N}=-2 s\left(1-N_{\bar{\Sigma}}\right)-h N_{g}-(p+\bar{p}) N_{\Psi}, \\
& \mathcal{A}_{L}^{N}=h\left(1-N_{\overline{\mathcal{H}}}\right)-2(h+s) N_{g}-2 p N_{\Psi}, \\
& \mathcal{A}_{R}^{N}=h\left(1-N_{\overline{\mathcal{H}}}\right)+2 s N_{g}-2 \bar{p} N_{\Psi},
\end{aligned}
$$

where $N_{g}=3$ is the number of generations of $\mathrm{SM}$ fermions. Here, $N_{\overline{\mathcal{H}}}, N_{\bar{\Sigma}}$ are respectively the number of
$\overline{\mathcal{H}}, \bar{\Sigma}$, while we take $N_{\mathcal{H}}=N_{\Sigma}=1$. In the minimal model, $N_{\overline{\mathcal{H}}}=N_{\bar{\Sigma}}=0, \bar{r}=r$ and $\bar{p}=p$.

The conditions for anomaly cancellation are given by

$$
\begin{aligned}
\mathcal{A}_{C}^{4 R} \equiv \mathcal{A}_{L}^{4 R} \equiv \mathcal{A}_{R}^{4 R} & \text { modulo } 2, \\
\mathcal{A}_{C}^{N} \equiv \mathcal{A}_{L}^{N} \equiv \mathcal{A}_{R}^{N} & \text { modulo } N .
\end{aligned}
$$

The anomaly is completely canceled if these are vanishing, while these can be canceled by the Green-Schwartz mechanism [49] if these are nonvanishing but have a universal value. In this paper, we will consider the minimal case $N_{\overline{\mathcal{H}}}=$ $N_{\bar{\Sigma}}=0$ and the next-to-minimal case $N_{\overline{\mathcal{H}}}=N_{\bar{\Sigma}}=1$.

\section{The axion quality}

There will be numerous higher dimensional operators which may explicitly break the PQ symmetry. In general, the $\theta$ angle will be shifted at tree-level by a PQ breaking term in the superpotential,

$$
W_{\mathrm{PQQ}} \supset \frac{1}{\Lambda^{k+2 l+m+n-3}} w_{0}^{k} \mathcal{H}^{2 l} P^{m} \bar{P}^{n},
$$

where $k, l, m, n$ are integers. Note, contributions from operators with $\overline{\mathcal{H}}^{2}$ cannot be the leading ones, since as long as $\Delta W \supset(P, \bar{P})^{2} \mathcal{H}^{2} / \Lambda$ in Eq. (10) is allowed for the $\mu / b$ term and we have $\langle\overline{\mathcal{H}}\rangle^{2}<w_{0} f_{\mathrm{PQ}}^{2} / \Lambda$, which is satisfied for the typical values of VEVs. The powers satisfy

$$
\begin{array}{cc}
2 k+m r+n \bar{r} \equiv 2 & \text { modulo } 4, \\
2 l h+m p+n \bar{p} \equiv 0 & \text { modulo } N .
\end{array}
$$

This term will affect the $\theta$ angle via the F-term potential and the soft SUSY breaking $A$-term. The leading PQ breaking in the F-term potential will be an interference term between the PQ conserving and breaking terms in the superpotential. Consider that the F-term VEVs will be

$$
F_{P}, F_{\bar{P}} \lesssim w_{0}^{2}, \quad F_{\mathcal{H}} \lesssim v_{H} w_{0}
$$

where $F_{\Phi}$ is an F-term of a superfield $\Phi=P, \bar{P}, \mathcal{H}$. The F-term of the Higgs field will depend on $v_{H}$ because $\mathcal{H}^{2}$ is a gauge singlet combination. We can show that

$$
V_{F} \supset \sum_{\Phi=P, \bar{P}, \mathcal{H}} F_{\Phi} \frac{\partial W_{\mathrm{PQ}}}{\partial \Phi} \lesssim w_{0} W_{\mathrm{PQ}}
$$

where the right-hand side corresponds to the A-term contribution. Hence, it is enough to confirm that $\Delta \theta$ from the A-term, 


$$
\begin{aligned}
\Delta \theta & \sim \frac{w_{0}^{1+k} v_{H}^{2 l} f_{\mathrm{PQ}}^{m+n}}{\Lambda_{\mathrm{QCD}}^{4} \Lambda^{k+2 l+m+n-3}} \\
\sim & 10^{63-13 k-32 l-8(m+n)} \times\left(\frac{w_{0}}{10^{5} \mathrm{GeV}}\right)^{1+k} \\
& \times\left(\frac{f_{\mathrm{PQ}}}{10^{10} \mathrm{GeV}}\right)^{m+n}\left(\frac{10^{18} \mathrm{GeV}}{\Lambda}\right)^{k+2 l+m+n-3}
\end{aligned}
$$

is sufficiently suppressed.

We set the Higgs VEV, $v_{H}=100 \mathrm{GeV}$ and the QCD scale in front of the axion potential, $\Lambda_{\mathrm{QCD}}=100 \mathrm{MeV}$. For $k=l=0, m+n \geq 10$ is typically required for $\Delta \theta<10^{-10}$ to solve the strong $C P$ problem. Clearly, $\mathbb{Z}_{4}^{R}$ alone cannot suppress the self-coupling of $P$ up to this order, and an additional symmetry such as the $\mathbb{Z}_{N}$ symmetry is necessary. Note that the PS breaking VEV cannot be the leading PQ breaking effect, since $\bar{S}^{c} S^{c}$ is a unique gauge singlet combination whose VEV is nonzero, but this is neutral under the discrete symmetries. Hence, $\langle P\rangle \sim\langle\bar{P}\rangle \sim f_{\mathrm{PQ}}$ will be the largest source of PQ breaking.

There might also be radiative corrections to the potential induced by explicit PQ breaking interactions. Let us consider a term in a superpotential,

$$
W_{\mathrm{PQ}} \supset \frac{w_{0}^{a} \mathcal{H}^{2 b} P^{c} \bar{P}^{d}\left(S^{c}, \bar{S}^{c}\right)^{e}}{\Lambda^{a+2 b+c+d+e-1}} \phi_{1} \phi_{2}
$$

where $\phi_{1}$ and $\phi_{2}$ can be any fields allowed by the discrete symmetries and they do not need to have a nonzero VEV. Here, $a, b, c, d, e$ are integers. The integers $a, e=0,1$ for the leading contributions. The PS breaking fields $S^{c}$ or $\bar{S}^{c}$ can appear in the leading contribution from e.g., $\phi_{1}=\mathcal{H}$, $\phi_{2}=Q$. There may be 1-loop corrections mediated by $\phi_{1}$, $\phi_{2}$ to the $\theta$ angle given by

$$
\begin{aligned}
\Delta \theta \sim & \frac{1}{16 \pi^{2}} \frac{w_{0}^{2+2 a} v_{H}^{4 b} f_{\mathrm{PQ}}^{2 c+2 d} v_{\mathrm{PS}}^{2 e}}{\Lambda_{\mathrm{CD}}^{4} \Lambda^{2 a+4 b+2 c+2 d+2 e-2}} \\
\sim & 10^{48-26 a-64 b-16(c+d)-4 e} \\
& \times\left(\frac{w_{0}}{10^{5} \mathrm{GeV}}\right)^{2+2 a}\left(\frac{f_{\mathrm{PQ}}}{10^{10} \mathrm{GeV}}\right)^{2 c+2 d}\left(\frac{10^{16} \mathrm{GeV}}{v_{\mathrm{PS}}}\right)^{2 e} \\
& \times\left(\frac{10^{18} \mathrm{GeV}}{\Lambda}\right)^{2 a+4 b+2 c+2 d+2 e-2} .
\end{aligned}
$$

For $a=b=0$ and $e=1, c+d \geq 4$ may be required to keep the axion quality, $\Delta \theta<10^{-10}$. Thus the radiative correction may not spoil the axion solution to the strong $C P$ problem if explicit PQ breaking terms are absent up to dimension-7 operators.

The PQ breaking in Kähler potential can also affect the $\theta$ angle. The leading contribution to the scalar potential will be given by

$$
\begin{aligned}
V_{\mathrm{PQ}} & \supset w_{0}^{2} K_{\mathrm{PQ}} \\
& \supset \frac{1}{\Lambda^{k+2 l+m+n-2}} w_{0}^{k+2} \mathcal{H}^{2 l} P^{m}\left(\bar{P}^{\dagger}\right)^{n} \\
& +\frac{1}{\Lambda^{a+2 b+c+d+e}} w_{0}^{a+2} \mathcal{H}^{2 b} P^{c}\left(\bar{P}^{\dagger}\right)^{d}\left(S^{c(\dagger)}, \bar{S}^{c(\dagger)}\right)^{e} \phi_{1}^{(\dagger)} \phi_{2}^{(\dagger)} .
\end{aligned}
$$

In the minimal model, we will assume that $\Delta W \supset w_{0} \mathcal{H}^{2}$ is allowed for the $\mu / b$-term, so $\mathcal{H}^{\dagger}$ has the same charge as $\mathcal{H}$ and will not induce a new PQ breaking operator. In the nonminimal model, operators involving $\left(\mathcal{H}^{\dagger}, \overline{\mathcal{H}}\right)^{2}$ cannot be the leading one for the same reason as those involving $\overline{\mathcal{H}}^{2}$ in the superpotential. Thus, the terms in Eq. (24) give the leading PQ violating effect from the Kähler potential. The shift in $\theta$ from the first term is estimated as

$$
\begin{aligned}
\Delta \theta & \sim \frac{w_{0}^{k+2} v_{H}^{2 l} f_{\mathrm{PQ}}^{m+n}}{\Lambda_{\mathrm{QCD}}^{4} \Lambda^{k+2 l+m+n-2}} \\
\sim & 10^{50-13 k-32 l-8(m+n)} \times\left(\frac{w_{0}}{10^{5} \mathrm{GeV}}\right)^{2+k}\left(\frac{f_{\mathrm{PQ}}}{10^{10} \mathrm{GeV}}\right)^{m+n} \\
& \times\left(\frac{10^{18} \mathrm{GeV}}{\Lambda}\right)^{k+2 l+m+n-2} .
\end{aligned}
$$

For $k=l=0, m+n \geq 8$ will be required for the axion quality. The loop correction mediated by $\phi_{1}^{(\dagger)}$ and $\phi_{2}^{(\dagger)}$ is estimated as

$$
\begin{aligned}
\Delta \theta \sim & \frac{w_{0}^{2 a+4} v_{H}^{4 b} f_{\mathrm{PQ}}^{2 c+2 d} v_{\mathrm{PS}}^{2 e}}{16 \pi^{2} \Lambda_{\mathrm{QCD}}^{4} \Lambda^{2 a+4 b+2 c+2 d+2 e}} \\
\sim & 10^{22-26 a-64 b-16(c+d)-4 e} \\
& \times\left(\frac{w_{0}}{10^{5} \mathrm{GeV}}\right)^{2 a+4}\left(\frac{f_{\mathrm{PQ}}}{10^{10} \mathrm{GeV}}\right)^{2 c+2 d}\left(\frac{v_{\mathrm{PS}}}{10^{16} \mathrm{GeV}}\right)^{2 e} \\
& \times\left(\frac{10^{18} \mathrm{GeV}}{\Lambda}\right)^{2 a+4 b+2 c+2 d}
\end{aligned}
$$

For $a=b=0, c+d=2$ and $e=0$ (1), the shift of $\theta$ is comparable to the experimental bound, $\Delta \theta \sim 10^{-10}\left(10^{-14}\right)$. It is much smaller for $c+d>2$.

\section{E. R-parity violation and proton stability}

Stability of the proton and LSP are not ensured in this model, because the discrete symmetries $\mathbb{Z}_{4}^{R} \times \mathbb{Z}_{N}$ are broken by the PQ fields. If all the PQ fields have even R-charge, R-parity remains unbroken. The LSP will be stable, but dimension-5 (or higher) baryon and lepton number violating operators would destabilize the proton. Although these may be highly suppressed as discussed in Refs. [27,28]. If $P$ and/or $\bar{P}$ have odd R-charge, $\mathbb{Z}_{4}^{R}$ symmetry is completely broken. Then R-parity might 
appear as an accidental symmetry, if the RPV operators are highly suppressed.

The $\mathbb{Z}_{N}$ symmetry is also completely broken by $f_{\mathrm{PQ}}$ if the charge of $P$ and/or $\bar{P}$ is not a divisor of $N$. In this model, the dimension- 4 RPV operators are induced from the operators,

$$
\begin{gathered}
Q \mathcal{H} S^{c}:(1,0), \quad Q \overline{\mathcal{H}} S^{c}:(1,-2 h), \\
Q Q^{c} Q S^{c}:(3,-2 h), \quad Q^{c} Q^{c} Q^{c} S^{c}:(3,4 s),
\end{gathered}
$$

after the PS breaking. Here, the values in the parentheses are charges under $\left(\mathbb{Z}_{4}^{R}, \mathbb{Z}_{N}\right)$. The first three operators violate lepton number, while the last one violates baryon number. The superpotential is given by

$$
\begin{aligned}
W_{\mathrm{RPV}}= & y_{L} Q \mathcal{H} S^{c}+\bar{y}_{L} Q \overline{\mathcal{H}} S^{c}+\kappa_{L} Q Q^{c} Q S^{c} \\
& +\kappa_{B} Q^{c} Q^{c} Q^{c} S^{c},
\end{aligned}
$$

where the coupling constants depend on certain combinations of $P, \bar{P}, w_{0}$ and $\mathcal{H}^{2}$ to be consistent with the discrete symmetry. The effective $\Delta L=1(\Delta B=1)$ Yukawa coupling $\lambda_{L}\left(\lambda_{B}\right)$ induced by those operators are given by

$\lambda_{L} \sim \max \left(\frac{y_{L} v_{\mathrm{PS}}}{w_{0}}, \frac{\bar{y}_{L} v_{\mathrm{PS}}}{w_{0}}, \kappa_{L} v_{\mathrm{PS}}\right), \quad \lambda_{B} \sim \kappa_{B} v_{\mathrm{PS}}$.

Here, we assume that the Yukawa coupling of $Q \mathcal{H} Q^{c}$ is $\mathcal{O}(1)$ and the higgsino mass is $\mathcal{O}\left(w_{0}\right)$. The bilinear $\Delta L=1$ Yukawa couplings are induced by rotating away the bilinear RPV terms by redefining Higgs and leptons. The proton decay constraints on the RPV operators are

$$
\lambda_{L} \lambda_{B} \lesssim 10^{-27} \times\left(\frac{m_{\tilde{f}}}{1 \mathrm{TeV}}\right)^{2},
$$

where $m_{\tilde{f}}$ is a sfermion mass.

The LSP will become unstable if there are sizable RPV effects. If the lepton number violation dominates RPV, the lifetime of a neutralino LSP via the three-body decay, $\chi \rightarrow \nu \ell \ell^{\dagger}$, where $\ell \ell^{\dagger}=e^{+} e^{-}, \nu \bar{\nu}$, is estimated as [50,51],

$\tau_{\chi} \sim \frac{1536 \pi^{3}}{g_{\nu \mathrm{Z}}^{4} N_{\chi \nu}^{2}} \frac{m_{\mathrm{Z}}^{4}}{m_{\chi}^{5}} \sim 1 \mathrm{~s} \times\left(\frac{0.1}{g_{\nu \mathrm{Z}}}\right)^{4}\left(\frac{10^{-14}}{N_{\chi \nu}}\right)^{2}\left(\frac{10^{4} \mathrm{GeV}}{m_{\chi}}\right)^{5}$.

Here, we consider the three-body decay through a Z-boson whose mass is set at $100 \mathrm{GeV}^{7}$ The decays through the EW boson dominate over those through sfermions, if the sfermions are heavier than $\mathcal{O}(10 \mathrm{TeV})$, see Eq. (33). $g_{\nu Z}$ is the coupling constant of the neutrinos to a Z-boson. The mass of the neutralino LSP is denoted by $m_{\chi} . N_{\chi \nu}$ is the

\footnotetext{
${ }^{7}$ The decay through a W-boson will have the same size.
}

mixing angle of the LSP and the neutrino whose size is estimated as

$$
N_{\chi \nu} \sim N_{\chi \tilde{H}} \times \max \left(\frac{y_{L} v_{\mathrm{PS}}}{w_{0}}, \frac{\bar{y}_{L} v_{\mathrm{PS}}}{w_{0}}, \frac{\kappa_{L} v_{\mathrm{PS}}}{16 \pi^{2}}\right),
$$

where $N_{\chi \tilde{H}}$ is the fraction of Higgsinos in the lightest neutralino $\chi$. The last one comes from mixing at the 1-loop level via the RPV Yukawa coupling. Here, the MSSM Yukawa coupling is set to 1 for simplicity. The LSP decays before BBN, i.e., $\tau_{\chi} \lesssim 1 \mathrm{~s}$, if $N_{\chi \nu} \gtrsim 10^{-14}$. On the other hand, the lifetime is longer than $\mathcal{O}\left(10^{24}\right.$ s) if $N_{\chi \nu} \lesssim 10^{-26}$, such that the neutralino LSP is the stable DM and its decay does not affect the cosmic microwave background [29].

If the baryon number violation is the dominant one, then the Higgsino-lepton mixing is negligible. Hence, the neutralino decays through squarks and its lifetime is estimated as

$$
\begin{aligned}
\tau_{\chi} & \sim \frac{1536 \pi^{3}}{g_{\chi \tilde{q}}^{2} \lambda_{B}^{2}} \frac{m_{\tilde{q}}^{4}}{m_{\chi}^{5}} \\
& \sim 1 \mathrm{~s} \times\left(\frac{0.1}{g_{\chi \tilde{q}}}\right)^{2}\left(\frac{10^{-9}}{\lambda_{B}}\right)^{2}\left(\frac{m_{\tilde{q}}}{10^{5} \mathrm{GeV}}\right)^{4}\left(\frac{10^{4} \mathrm{GeV}}{m_{\chi}}\right)^{5}
\end{aligned}
$$

where $g_{\chi \tilde{q}}$ is a coupling constant for the quark-squark-LSP interaction and $m_{\tilde{q}}$ is a squark mass. The LSP decays before BBN if $\lambda_{\mathrm{B}} \gtrsim 10^{-9}$, while the LSP is a stable and invisible DM particle if $\lambda_{\mathrm{B}} \lesssim 10^{-21}$.

Proton decay may also be mediated by dimension- 5 operators,

$$
Q Q Q Q:(0,-4 h-4 s), \quad Q^{c} Q^{c} Q^{c} Q^{c}:(0,4 s),
$$

in the superpotential and/or dimension- 6 operator,

$$
Q^{\dagger} Q^{\dagger} Q^{c} Q^{c}:(0,2 h+4 s),
$$

in the Kähler potential. The effective cut-off scale for the dimension-5 and -6 operators, $\Lambda_{5}$ and $\Lambda_{6}$, should be larger than $\mathcal{O}\left(10^{27}\right)$ and $\mathcal{O}\left(10^{15}\right) \mathrm{GeV}$, respectively. These operators will be sufficiently suppressed by the discrete symmetries. Note, however, that the dimension-5 and -6 operators, as well as the RPV operators, might also be generated after integrating out vectorlike triplets which are much lighter than the GUT scale. Clearly we need to check that these are also suppressed.

Let us consider the vectorlike triplets $(T, \bar{T})$, which are in $(\Psi, \bar{\Psi}),\left(\bar{\Psi}^{c}, \Psi^{c}\right)$ or $(\bar{\Sigma}, \Sigma)$, with a superpotential,

$$
\begin{gathered}
W \supset m_{T} \bar{T} T+T\left(\mu_{\bar{T}} \overline{\mathcal{Q}}_{1}^{T}+\lambda_{\bar{T}} \overline{\mathcal{Q}}_{2}^{T}+\kappa_{\bar{T}} \overline{\mathcal{Q}}_{3}^{T}\right) \\
+\bar{T}\left(\mu_{T} \mathcal{Q}_{1}^{T}+\lambda_{T} \mathcal{Q}_{2}^{T}+\kappa_{T} \mathcal{Q}_{3}^{T}\right),
\end{gathered}
$$


where $\mathcal{Q}_{k}^{T}$ and $\overline{\mathcal{Q}}_{k}^{T}$ are composed of $\left\{Q, Q^{c}, \Sigma, \bar{\Sigma}\right\}$ for $(T, \bar{T})=\left(\Psi_{q}, \bar{\Psi}_{q}\right),\left(\bar{\Psi}_{q}^{c}, \Psi_{q}^{c}\right)$, and $\left\{Q, Q^{c}\right\}$ for $(T, \bar{T})=$ $(\sigma, \bar{\sigma})$. Here, integer $k$ represents the mass dimension of $\mathcal{Q}_{k}^{T}$ and $\overline{\mathcal{Q}}_{k}^{T}$. $\Psi_{q}\left(\bar{\Psi}_{q}\right)$ and $\bar{\Psi}_{q}^{c}\left(\Psi_{q}^{c}\right)$ are color (anti-)triplets in $\Psi$ $(\bar{\Psi})$ and $\bar{\Psi}^{c}\left(\Psi^{c}\right)$, respectively. The coupling constants depend on the nonzero VEVs of the fields. After integrating out the vectorlike triplets, we have

$$
\begin{aligned}
W \supset & \frac{1}{m_{T}}\left(\mu_{T} \mu_{\bar{T}} Q_{1}^{T} \bar{Q}_{1}^{T}+\mu_{T} \lambda_{\bar{T}} \mathcal{Q}_{1}^{T} \overline{\mathcal{Q}}_{2}^{T}+\lambda_{T} \mu_{\bar{T}} \mathcal{Q}_{2}^{T} \overline{\mathcal{Q}}_{1}^{T}\right. \\
& \left.+\mu_{T} \kappa_{\bar{T}} \mathcal{Q}_{1}^{T} \overline{\mathcal{Q}}_{3}^{T}+\lambda_{T} \lambda_{\bar{T}} \mathcal{Q}_{2}^{T} \overline{\mathcal{Q}}_{2}^{T}+\kappa_{T} \lambda_{\bar{T}} \mathcal{Q}_{3}^{T} \overline{\mathcal{Q}}_{1}^{T}\right),
\end{aligned}
$$

where we omit the higher-dimensional operators. The mass mixing effects of the first term may be sufficiently suppressed as will be shown in explicit examples in Sec. III. The latter two terms in the first line may induce the RPV Yukawa couplings and the second line may include the dimension-5 operators. Without discussing details, the proton will be stable if

$$
\begin{gathered}
\max \left(\frac{\mu_{T} \bar{\lambda}_{T}}{m_{T}}, \frac{\lambda_{T} \bar{\mu}_{T}}{m_{T}}\right) \ll 10^{-17} \times\left(\frac{10^{-10}}{\lambda_{L}}\right), \\
\max \left(\frac{\mu_{T} \bar{\kappa}_{T}}{m_{T}}, \frac{\lambda_{T} \bar{\lambda}_{T}}{m_{T}}, \frac{\kappa_{T} \bar{\mu}_{T}}{m_{T}}\right) \ll\left(10^{27} \mathrm{GeV}\right)^{-1} .
\end{gathered}
$$

Since we will find $\mu_{T} / m_{T} \lesssim 1$ in our examples, $\max \left(\lambda_{T}, \lambda_{\bar{T}}\right) \ll 10^{-17}$ and $\max \left(\kappa_{T}, \kappa_{\bar{T}}\right) \ll 10^{-27} \mathrm{GeV}^{-1}$ are sufficient conditions for the proton stability.

In the Kähler potential, the sizable dimension- 6 operator could be induced by integrating out the color triplets from

$$
\begin{aligned}
K \supset & \frac{1}{\Lambda}\left(Q^{c} Q^{c}\right)^{\dagger}\left(\zeta_{\Sigma} \Sigma+\frac{\zeta_{\Psi}}{\Lambda} S^{c} \Psi^{c}\right) \\
& +\frac{1}{\Lambda}\left(\zeta_{\Sigma}^{c} \bar{\Sigma}+\frac{\zeta_{\Psi}^{c}}{\Lambda} \bar{S}^{c} \bar{\Psi}^{c}\right)^{\dagger} Q Q
\end{aligned}
$$

where $\zeta_{\Phi}^{(c)}, \Phi=\Sigma, \Psi, \Psi^{c}$ are coupling constants implicitly depending on the nonzero VEVs of gauge singlet combinations. The dimension- 6 operators will be generated by integrating out scalar components in the vectorlike fields, together with Yukawa couplings,

$$
\begin{aligned}
W \supset & \lambda_{Q^{c} \Sigma Q^{c}} Q^{c} \Sigma Q^{c}+\lambda_{Q \bar{\Sigma} Q} Q \bar{\Sigma} Q+\kappa_{Q^{c} Q^{c} S^{c} \Psi^{c}} Q^{c} Q^{c} S^{c} \Psi^{c} \\
& +\kappa_{Q Q \overline{S^{c}} \bar{\Psi}^{c}} Q Q \bar{S}^{c} \bar{\Psi}^{c} .
\end{aligned}
$$

Note that $\lambda_{Q^{c} \Sigma Q^{c}}\left(\lambda_{Q \bar{\Sigma} Q}\right)$ is a part of $\lambda_{\bar{\sigma}}\left(\lambda_{\sigma}\right)$ in Eq. (36) and

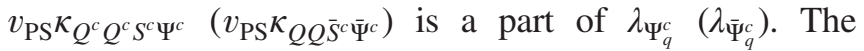
dimension- 6 operators arise as

$$
\begin{aligned}
\int d^{4} \theta K \supset & \frac{1}{\Lambda}\left(Q^{c} Q^{c}\right)^{\dagger}\left(\zeta_{\Sigma} F_{\bar{\sigma}}+\frac{\zeta_{\Psi}}{\Lambda} v_{\mathrm{PS}} F_{\Psi_{q}^{c}}\right) \\
+ & \frac{1}{\Lambda}\left(\zeta_{\Sigma}^{c} F_{\sigma}+\frac{\zeta_{\Psi}^{c}}{\Lambda} v_{\mathrm{PS}} F_{\bar{\Psi}_{q}^{c}}\right)^{\dagger} Q Q \\
\rightarrow & \left(\frac{\zeta_{\Sigma} \lambda_{Q \bar{\Sigma} Q}}{m_{\sigma} \Lambda}+\frac{\zeta_{\Psi} v_{\mathrm{PS}}^{2} \kappa_{Q Q \bar{S}^{c} \bar{\Psi}^{c}}}{m_{\Psi^{c}} \Lambda^{2}}\right)\left(u^{c} e^{c}\right)^{\dagger} q q \\
+ & \left(\frac{\zeta_{\Sigma}^{c} \lambda_{Q^{c} \Sigma Q^{c}}}{m_{\sigma} \Lambda}+\frac{\zeta_{\Psi}^{c} v_{\mathrm{PS}}^{2} \kappa_{Q^{c} Q^{c} S^{c} \Psi^{c}}}{m_{\Psi^{c}} \Lambda^{2}}\right)\left(u^{c} d^{c}\right)^{\dagger} q \ell,
\end{aligned}
$$

where $Q, Q^{c}$ are fermionic components of the superfields of the same symbols. Here, $F_{\Phi}, \Phi=\sigma, \bar{\sigma}, \Psi_{q}^{c}, \bar{\Psi}_{q}^{c}$, are the F-terms of color (anti-)triplets in the vectorlike fields. The scalar fields in the vectorlike fields are integrated out in the second equality. For example, the F-term of $\sigma$ in $\bar{\Sigma}$ is given by

$$
F_{\sigma} \sim-m_{\sigma} \bar{\sigma}^{*} \sim \frac{\lambda_{Q^{c} \Sigma Q^{c}} u^{c} d^{c}}{m_{\sigma}} .
$$

Thus, the sufficient condition is $\max \left(\lambda_{Q^{c} \Sigma Q^{c}}, \lambda_{Q \bar{\Sigma} Q}\right) \ll 10^{-7}$

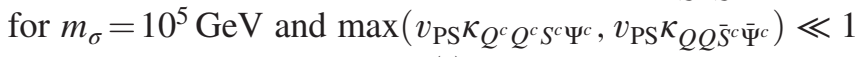
for $m_{\Psi}=10^{10} \mathrm{GeV}$, since $\zeta_{\Sigma, \Psi}^{(c)}<\mathcal{O}(1)$. These are much weaker constraints than those from the superpotential. Operators more suppressed by $\Lambda$ will always be sufficiently small because the effective cut-off scale will be larger than $\Lambda$ as far as $\mu_{T}<1 \mathrm{GeV}, \lambda_{T}<1$ and $\kappa_{T}<1 \mathrm{GeV}^{-1}$, which are clearly satisfied in our examples.

These sufficient conditions in the superpotential and Kähler potential are satisfied in our examples shown in the next section, so we will not discuss any more details of proton decay in this paper.

\section{MODEL EXAMPLES}

\section{A. Minimal model: High-quality LSP}

Let us first consider the minimal model with $N_{\overline{\mathcal{H}}}=$ $N_{\bar{\Sigma}}=N_{\bar{P}}=0, N_{\Psi}=1$ and $s=0$. The anomaly cancellation for $\mathbb{Z}_{N}$ implies,

$$
-3 h \equiv-5 h \equiv h \quad \text { modulo } N .
$$

The solution is $h=0$ if $N$ is odd, and is $h \equiv 0$ modulo $N / 2$ if $N$ is even. Under this condition, $\mathcal{H}^{2}$ is neutral under the discrete symmetries. The mass term itself is forbidden by the $\mathbb{Z}_{4}^{R}$ symmetry, but the $\mu$-term will be generated after SUSY breaking by $W \supset w_{0} \mathcal{H}^{2}$. In addition, the $b$-term will be generated from this term after SUSY breaking. Therefore, the condition (3) is satisfied whenever the condition (1) is satisfied.

For concreteness, we shall choose the charges

$$
N=5, \quad r=p=r_{\Psi}=1, \quad h=p_{\Psi}=0 .
$$


TABLE III. The charges under the discrete symmetries and the accidental $U(1)_{\mathrm{PQ}}$ in the minimal model.

\begin{tabular}{lcccccccccccc}
\hline \hline & $\mathcal{H}$ & $Q$ & $Q^{c}$ & $X$ & $S^{c}$ & $\bar{S}^{c}$ & $\Sigma$ & $\bar{\Psi}$ & $\Psi$ & $\Psi^{c}$ & $\bar{\Psi}^{c}$ & $P$ \\
\hline $\mathbb{Z}_{4 R}$ & 0 & 1 & 1 & 2 & 0 & 0 & 2 & 0 & 1 & 1 & 0 & 1 \\
$\mathbb{Z}_{5}$ & 0 & 0 & 0 & 0 & 0 & 0 & 0 & 4 & 0 & 0 & 4 & 1 \\
$U(1)_{\mathrm{PQ}}$ & 0 & 0 & 0 & 0 & 0 & 0 & 0 & -1 & 0 & 0 & -1 & 1 \\
\hline \hline
\end{tabular}

The charges of the fields are listed in Table III. With this charge assignment, $\Psi\left(\Psi^{c}\right)$ have the same charge as $Q\left(Q^{c}\right)$, so that these are like a fourth generation of the MSSM (s)fermions, but with vectorlike masses of $\mathcal{O}\left(f_{\mathrm{PQ}}\right)$, see Eq. (8). It is clear that the Yukawa coupling $Q \mathcal{H} Q^{c}$ will induce the decays of the vectorlike particles, and thus this model satisfies the condition (4). Gauge coupling unification is preserved and condition (5) is satisfied, since all the triplets in $S^{c}, \bar{S}^{c}$ and $\Sigma$ have masses of $\mathcal{O}\left(v_{\mathrm{PS}}\right)$. There is a fourth family of vectorlike fields with mass of $\mathcal{O}\left(f_{\mathrm{PQ}}\right)$ and the MSSM particles have mass less than the SUSY breaking scale.

We can find an accidental anomalous $U(1)_{\mathrm{PQ}}$ symmetry whose charges are shown in the last row of Table III. The MSSM particles cannot carry $U(1)_{\mathrm{PQ}}$ charge to be consistent with the PS superpotential,

$$
W_{\mathrm{PS}} \supset \frac{1}{\Lambda}\left(\bar{S}^{c} Q^{c}\right)^{2}+Q \mathcal{H} Q^{c}+w_{0} \mathcal{H}^{2}
$$

Hence, only the vectorlike quarks carry the $U(1)_{\mathrm{PQ}}$ charge, so the model has the KSVZ axion. The PQ breaking superpotential is given by

$$
W_{P Q}=\frac{P^{10}}{\Lambda^{7}}+\frac{P^{5}}{\Lambda^{4}} Q^{c} \bar{S}^{c}+\frac{P^{5}}{\Lambda^{5}} \mathcal{H} Q S^{c}+\cdots .
$$

These terms induce the shift in the $\theta$ angle by $\sim 10^{-17}, 10^{-32}$ and $10^{-36}$, respectively, and thus the PQ symmetry is so high quality that it solves the strong $C P$ problem. All the operators which can contribute to $\Delta \theta$ are listed in Table VI of Appendix B. In this model, the axion domain-wall number is $N_{\mathrm{DW}}=4$ and is not unity. However, the domainwall is unstable due to the explicit PQ breaking effects, thus it would not cause a cosmological problem [52]. ${ }^{8}$

The standard R-parity violations are extremely suppressed due to the discrete symmetry. In the minimal model, the lowest order for which $P$ can couple to the RPV operators is $P^{5}$, because none of the operators in Eqs. (27) have $\mathbb{Z}_{5}$ charge. In fact, the bilinear RPV term is of order $\sim 10^{-24} \mathrm{GeV}$ and the lepton number violating Yukawa couplings are of order $\sim 10^{-55}$ in this model, and

\footnotetext{
${ }^{8}$ It would also be solved by the dynamics of multiple scalar fields [53].
}

thus $\lambda_{L} \sim 10^{-29}$ for $w_{0} \sim 10^{5} \mathrm{GeV}$. The coefficient of dimension-4 baryon number violating operator is of order $10^{-55}$. This is clearly sufficiently small to make the lifetime of the neutralino LSP longer than the age of the universe, see Eq. (32). For the same reason, the proton lifetime is extremely long. Therefore, R-parity exists very precisely in this model. The full list of operators and their typical values relevant to the proton decays are shown in Table VII of Appendix B. This conclusion will not be changed since the MSSM fields do not carry $\mathbb{Z}_{N}$ charges as is required by the anomaly cancellation in the minimal model.

The stable LSP may or may not be a problem. The LSP is known to be an attractive candidate for the DM if the neutralino masses are in the suitable range, e.g., Higgsino $\sim 1 \mathrm{TeV}$ and the conventional thermal freeze-out scenario is working $[54,55]$. However, the LSP tends to overclose the universe in high-scale SUSY scenarios. In particular, the nonthermal production from the gravitino and/or moduli often overproduce the LSP $[30,32,56]$. This overproduction problem could be solved if the LSP is much lighter than the $\mathrm{TeV}$ scale. An axino with a mass $\lesssim \mathcal{O}(\mathrm{keV})$, is a candidate for such a particle [57-59] if it is sufficiently stable.

We shall discuss a case of $\mathcal{O}(\mathrm{keV})$ axino LSP. The axino mixes with neutrinos by the RPV effects. Defining the axion superfield $A$ via

$P=f_{\mathrm{PQ}} e^{A / f_{\mathrm{PQ}}}, \quad A=\frac{1}{\sqrt{2}}(s+i a)+\sqrt{2} \theta \tilde{a}+\theta^{2} F_{A}$,

where $s, a$ and $\tilde{a}$ are saxion, axion and axino, respectively. $F_{A}$ is the F-term of the superfield $A$. Integrating out the right-handed neutrinos, we find

$$
\begin{aligned}
W & \supset \frac{1}{M_{R}}\left(\ell H_{u}+\frac{v_{\mathrm{PS}} P^{5}}{\Lambda^{4}}\right)^{2} \\
& \supset \frac{f_{\mathrm{PQ}}^{4}}{v_{\mathrm{PS}} \Lambda^{3}}\left(f_{\mathrm{PQ}} H_{u}^{0} \nu+v_{H} A \nu+\frac{v_{H}}{f_{\mathrm{PQ}}} A A \nu\right),
\end{aligned}
$$

where $\mathcal{O}(1)$ coefficients are omitted and $M_{R}$ is replaced by using Eq. (5). The first two terms induce the RPV Higgsino-neutrino and axino-neutrino mixing, respectively. These could affect the neutrino mass by

$$
\begin{aligned}
\delta m_{\nu} \sim & \left(\frac{v_{H} f_{\mathrm{PQ}}^{4}}{v_{\mathrm{PS}} \Lambda^{3}}\right)^{2} \max \left(\frac{f_{\mathrm{PQ}}^{2}}{w_{0}}, \frac{v_{H}^{2}}{m_{\tilde{a}}}\right) \\
\sim & 10^{-32} \mathrm{eV} \times\left(\frac{10^{16} \mathrm{GeV}}{v_{\mathrm{PS}}}\right)^{2}\left(\frac{f_{\mathrm{PQ}}}{10^{10} \mathrm{GeV}}\right)^{8} \\
& \times\left(\frac{10^{18} \mathrm{GeV}}{\Lambda}\right)^{6}\left(\frac{\max \left(f_{\mathrm{PQ}}^{2} / w_{0}, v_{H}^{2} / m_{\tilde{a}}\right)}{10^{15} \mathrm{GeV}}\right),
\end{aligned}
$$

where $m_{\tilde{a}}$ is the axino mass and Higgsino mass is assumed to be $\mathcal{O}\left(w_{0}\right)$. Thus the mixing will not affect neutrino masses. 
The axino will dominantly decay by $\tilde{a} \rightarrow \nu a$ or $\tilde{a} \rightarrow \nu \ell \ell^{\dagger}$, where $\ell \ell^{\dagger}=e^{+} e^{-}, \nu \nu$, as discussed in Refs. [60-63]. The decay to electrons are allowed when $m_{\tilde{a}}>2 m_{e}$. The first decay mode is induced by the last term in Eq. (50) and the lifetime via this mode is estimated as,

$$
\begin{aligned}
\tau_{\tilde{a} \rightarrow \nu a} \sim & \frac{16 \pi}{m_{\tilde{a}}} \frac{\Lambda^{6} v_{\mathrm{PS}}^{2}}{v_{H}^{2} f_{\mathrm{PQ}}^{6}} \\
\sim & 10^{52} \text { years } \times\left(\frac{1 \mathrm{keV}}{m_{\tilde{a}}}\right)\left(\frac{\Lambda}{10^{18} \mathrm{GeV}}\right)^{6} \\
& \times\left(\frac{v_{\mathrm{PS}}}{10^{16} \mathrm{GeV}}\right)^{2}\left(\frac{10^{10} \mathrm{GeV}}{f_{\mathrm{PQ}}}\right)^{6} .
\end{aligned}
$$

The second decay mode is similar to the neutralino decay and can be estimated from Eq. (31) with formally replacing $\chi \rightarrow \tilde{a}$,

$$
\begin{aligned}
\tau_{\tilde{a} \rightarrow \nu \ell \ell} \sim & \frac{1536 \pi^{3}}{g_{\nu Z}^{4}} \frac{\Lambda^{6} v_{\mathrm{PS}}^{2} v_{H}^{2}}{f_{\mathrm{PQ}}^{8} m_{\tilde{a}}^{3}} \\
\sim & 10^{59} \text { years } \times\left(\frac{0.1}{g_{\nu Z}}\right)^{4}\left(\frac{1 \mathrm{keV}}{m_{\tilde{a}}}\right)^{3}\left(\frac{\Lambda}{10^{18} \mathrm{GeV}}\right)^{6} \\
& \times\left(\frac{v_{\mathrm{PS}}}{10^{16} \mathrm{GeV}}\right)^{2}\left(\frac{10^{10} \mathrm{GeV}}{f_{\mathrm{PQ}}}\right)^{8}
\end{aligned}
$$

Here, the neutrino-axino mixing comes from the second term in Eq. (50). These are both much longer the age of universe, and thus the axino will be a DM particle if its mass is of $\mathcal{O}(\mathrm{keV})$ and it is the LSP.

Another way to resolve the overproduction problem is that the LSP is unstable due to sizable RPV and it does not contribute to the DM. We will show an example with sizable RPV in the next section.

\section{B. RPV model: Low-quality LSP}

Let us consider the model with the two PQ fields $P, \bar{P}$ and $N_{\overline{\mathcal{H}}}=N_{\bar{\Sigma}}=1$. The new fields $\overline{\mathcal{H}}$ and $\bar{\Sigma}$ are mandatory for $h \neq 0$, because there is no such solution for the anomaly condition in the model only with $\bar{P}$. The anomaly cancellation conditions are given by

$$
\begin{array}{r}
r+\bar{r} \equiv 0 \quad \text { modulo } 2 \\
-3 h-p-\bar{p} \equiv-6(h+s)-2 p \equiv 6 s-2 \bar{p} \quad \operatorname{modulo} N
\end{array}
$$

for $\mathbb{Z}_{4}^{R}, \mathbb{Z}_{N}$, respectively.

In this section, we shall show an example which violates R-parity such that the LSP is unstable and decays before BBN. Let us first consider the RPV effects caused by the RPV Yukawa couplings. Note that the RPV by $Q \mathcal{H} S^{c}$ can not be sizable because it does not have $\mathbb{Z}_{N}$ charge, see
Eq. (27) and Table I. ${ }^{9}$ The sufficiently large RPV operators are induced if either of the following operators are allowed:

$$
\begin{gathered}
P Q S^{c} Q Q^{c}:(3+r,-2 h+p), \\
P Q^{c} Q^{c} Q^{c} S^{c}:(3+r, 4 s+p),
\end{gathered}
$$

These operators are allowed by $\mathbb{Z}_{4}^{R}$ if $r=3$. The first one is allowed if $p=2 h$ and the second one is allowed if $p=-4 s$. However, $p=-4 s$ is not phenomenologically viable since there also exists an operator $P S^{c} \bar{\Sigma} Q^{c}$ which induces too large a mass for the down quark $d^{c}$ of $\mathcal{O}\left(v_{\mathrm{PS}} f_{\mathrm{PQ}} / \Lambda\right)$. The same conclusion holds for $\bar{P}$, and thus the sizable RPV is realized when $P$ or $\bar{P}$ has charge $(3,2 h)$. Since the operator $w_{0} Q \overline{\mathcal{H}} S^{c}$ has charge $(3,-2 h), w_{0} P Q \overline{\mathcal{H}} S^{c}\left(w_{0} \bar{P} Q \overline{\mathcal{H}} S^{c}\right)$ is accompanied with $P Q S^{c} Q Q^{c}\left(\bar{P} Q S^{c} Q Q^{c}\right)$.

In the nonminimal model, the Higgsino masses are always generated by $W \supset w_{0} \mathcal{H} \overline{\mathcal{H}}$. However, the SUSY breaking $b$-term, $V \supset b_{h} H_{u} H_{d}$, is missing. For the $b$-term, $(P, \bar{P})^{2} \mathcal{H}^{2}$ should exist in the superpotential. We need two PQ fields for the sizable RPV and $b$-term, since $P^{2} \mathcal{H}^{2}$ is forbidden if $P$ has charge $(3,2 h)$.

Table IV shows four cases which realize both sizable RPV interaction and $b$-term. The PQ field $P$ induces the RPV in cases (I) and (II), while $\bar{P}$ does in cases (III) and (IV). The $b$-term is realized by $\bar{P}^{2} \mathcal{H}^{2}$ in the case (I), $P^{2} \mathcal{H}^{2}$ in the case (III) and $P \bar{P} \mathcal{H}^{2}$ in the cases (II) and (IV). The PQ charges are determined to be consistent with interactions in $W_{\mathrm{PS}}, W_{\mathrm{PQ}}$ and the operators for the sizable RPV and $b$-term. The axion domain wall number, $N_{\mathrm{DW}}$ is listed in the last row. For $N_{g}=3$ and $N_{\Psi}=1, N_{\mathrm{DW}}=8$ in the cases (I) and (III), while $N_{\mathrm{DW}}=2$ in the cases (II) and (IV).

The $\mathbb{Z}_{N}$ charge should be chosen such that all the unwanted operators are forbidden by $\mathbb{Z}_{N}$ and satisfy the anomaly cancellation condition Eq. (55). Also $2 h+4 s \not \equiv 0$ is required so that $p=2 h \neq-4 s$, as discussed earlier. In addition, for proton stability, if $2 h+4 s \equiv 0$ then both $P Q^{c} Q^{c} Q^{c} S^{c}$, which induces baryon number violation, and $P Q^{2} Q^{c} S^{c}$ which is lepton number violating are allowed. There are various explicit PQ breaking operators discussed in Sec. II D. In our model search, we set reference values of the scales at

$$
\begin{aligned}
\Lambda & =10^{18} \mathrm{GeV}, \quad v_{\mathrm{PS}}=10^{16} \mathrm{GeV}, \quad f_{\mathrm{PQ}}=10^{10} \mathrm{GeV}, \\
w_{0} & =10^{5} \mathrm{GeV}, \quad v_{H}=10^{2} \mathrm{GeV} .
\end{aligned}
$$

With these values, we require that the PQ breaking at the tree-level, Eqs. (21) and (25), are forbidden such that $\Delta \theta \leq 10^{-10}$. We also require that the quartic PQ breaking

\footnotetext{
${ }^{9}$ Another bilinear RPV becomes moderately large if $(P, \bar{P})^{3} Q \overline{\mathcal{H}} S^{c}$ or $w_{0}(P, \bar{P}) Q \overline{\mathcal{H}} S^{c}$ is allowed. We do not find any advantage in the first case as discussed in later. The condition to have the latter is the same as that to have the RPV Yukawa couplings.
} 
TABLE IV. The charges consistent with the sizable RPV and $b$-term. $\mathrm{PQ}_{\Phi}$ is the PQ charge of a field $\Phi$ normalized such that the minimal charge of $P, \bar{P}$ and $\mathcal{H}$ is unity. The PQ charge of $Q^{c}$ is zero for the Majorana neutrino mass and that of $\overline{\mathcal{H}}$ is opposite to $\mathcal{H}$.

\begin{tabular}{lcccccccccrr}
\hline \hline & RPV & $b$-term & $r$ & $\bar{r}$ & $p$ & $\bar{p}$ & $\mathrm{PQ}_{P}$ & $\mathrm{PQ}_{\bar{P}}$ & $\mathrm{PQ}_{\mathcal{H}}$ & $\mathrm{PQ}_{Q}$ & $N_{\mathrm{DW}}$ \\
\hline I & $P Q^{2} Q^{c} S^{c}$ & $\bar{P}^{2} \mathcal{H}^{2}$ & 3 & 1 & $2 h$ & $-h$ & -2 & 1 & -1 & 1 & $2\left|N_{g}+N_{\Psi}\right|$ \\
II & $P Q^{2} Q^{c} S^{c}$ & $\bar{P} P \mathcal{H}^{2}$ & 3 & 3 & $2 h$ & $-4 h$ & 2 & -4 & 1 & -1 & $2\left|2 N_{\Psi}-N_{g}\right|$ \\
III & $\bar{P} Q^{2} Q^{c} S^{c}$ & $P^{2} \mathcal{H}^{2}$ & 1 & 3 & $-h$ & $2 h$ & 1 & -2 & -1 & 1 & $2\left|N_{g}+N_{\Psi}\right|$ \\
IV & $\bar{P} Q^{2} Q^{c} S^{c}$ & $\bar{P} P \mathcal{H}^{2}$ & 3 & 3 & $-4 h$ & $2 h$ & -4 & 2 & 1 & -1 & $2\left|2 N_{\Psi}-N_{g}\right|$ \\
\hline \hline
\end{tabular}

TABLE V. The charges under the discrete symmetries $\mathbb{Z}_{4}^{R} \times \mathbb{Z}_{15}$ and an accidental $U(1)_{\mathrm{PQ}}$ symmetry in our example.

\begin{tabular}{lrrrrrrrrrrrrrrrr}
\hline \hline & $\mathcal{H}$ & $Q$ & $Q^{c}$ & $X$ & $S^{c}$ & $\bar{S}^{c}$ & $\Sigma$ & $\bar{\Sigma}$ & $\overline{\mathcal{H}}$ & $\bar{\Psi}$ & $\Psi$ & $\Psi^{c}$ & $\bar{\Psi}^{c}$ & $P$ & $\bar{P}$ \\
\hline $\mathbb{Z}_{4 R}$ & 0 & 1 & 1 & 2 & 0 & 0 & 2 & 2 & 0 & 2 & 1 & 1 & 2 & 3 & 3 \\
$\mathbb{Z}_{15}$ & 1 & 11 & 3 & 0 & 3 & 12 & 9 & 6 & 14 & 6 & 13 & 3 & 10 & 11 & 2 \\
$U(1)_{\mathrm{PQ}}$ & 1 & -1 & 0 & 0 & 0 & 0 & 0 & 0 & -1 & 3 & 1 & 0 & -2 & -4 & 2 \\
\hline \hline
\end{tabular}

combinations of $(P, \bar{P})^{2}(\mathcal{H}, \overline{\mathcal{H}})^{2}$ and $(P, \bar{P})^{2}(\Sigma, \bar{\Sigma})^{2}$ are forbidden to suppress PQ breaking via the 1-loop effects, see Eq. (23). For gauge coupling unification, $4 s \not \equiv 0$ modulo $N$ is required to keep the triplets in the sextets massless at $\mathcal{O}\left(v_{\mathrm{PS}}\right)$. Although these are still necessary conditions for the fully viable model, we can find solutions of these conditions only for $N=13$ in the case (II) or $N=15$ in all the four cases when $N \leq 16$ and $N_{\Psi}=1 .^{10}$

We shall study a solution, $(h, s)=(1,3)$, with $N=15$ in the case (IV). ${ }^{11}$ The charges of the fields are shown in Table V. A complete list of the possibly dangerous operators and their sizes are shown in Appendix B. We discuss operators important for phenomenology in the main text. The charges of vectorlike fields are chosen such that the Yukawa interactions,

$$
W_{\text {decay }}=\Psi \overline{\mathcal{H}} Q^{c}+Q \mathcal{H} \Psi^{c},
$$

are allowed by the symmetry so that the vectorlike fields decay quickly. The model with $W_{\text {decay }} \supset \Psi \mathcal{H} Q^{c}$ instead of $\Psi \overline{\mathcal{H}} Q^{c}$ also allows an exotic mass term $\bar{\Psi} \mathcal{H} \bar{S}^{c}:(2,0)$ which gives too large a mass term for $H_{u} \Psi_{\ell}$, where $\Psi_{\ell}$ is the leptonic component of the vectorlike field $\Psi$. The vectorlike triplets from the sextets will decay via $W \supset w_{0} Q^{c} \Sigma Q^{c}$

\footnotetext{
${ }^{10}$ We can find solutions for $N_{\Psi}=2$ when $N=11,15$, $N=13,15, N=15$ and $N=13,15$ in the case (I), (II), (III) and (IV), respectively. We did the same search for the RPV via $(P, \bar{P})^{3} Q \overline{\mathcal{H}} S^{c}$ in a case of $N_{\Psi}=1$, but we find setups consistent with these conditions only for $N \geq 14$. We will not study these cases.

${ }^{11}$ For $N=13$ in the case (II), $K_{\mathrm{PQ}} \supset P \bar{P}^{\dagger} \bar{\Sigma}^{2}$ is always allowed in the Kähler potential after imposing the anomaly condition. This induces $\Delta \theta \sim 10^{-10}$ which is marginal for the quality problem.
}

which is allowed by the symmetry independent of the charges $(h, s)$. The lifetime of the antitriplet $\bar{\sigma}$ by this interaction is estimated as

$\tau_{\sigma} \sim \frac{16 \pi \Lambda^{2}}{w_{0}^{3}} \sim 0.01 \mathrm{~s} \times\left(\frac{\Lambda}{10^{18} \mathrm{GeV}}\right)^{2}\left(\frac{10^{5} \mathrm{GeV}}{w_{0}}\right)^{3}$,

where the vectorlike triplet mass is set at $w_{0}$. The triplet $\sigma$ mixes with $\bar{\sigma}$ by the mass term of $\mathcal{O}\left(w_{0}\right)$. Thus the triplets will decay before BBN if the SUSY scale is as high as $100 \mathrm{TeV}$, and condition (4) is satisfied.

There are four Higgs doublets at the SUSY breaking scale whose mass terms are given by $W \supset f_{\mathrm{PQ}}^{2} \mathcal{H}^{2} / \Lambda+w_{0} \mathcal{H} \overline{\mathcal{H}}$. The $b$-term, $V_{h} \supset b_{h} H_{u} H_{d}$, will be generated after SUSY breaking from the first term. Therefore, both $\mu$ - and $b$-problems are solved in this model and the condition (3) is satisfied. Note that we invoke a fine-tuning of $\mathcal{O}\left(w_{0}^{2} / v_{H}^{2}\right) \sim \mathcal{O}\left(10^{6}\right)$ as usual in high-scale SUSY breaking scenarios. More details of the Higgs potential with $\overline{\mathcal{H}}$ is discussed in Appendix A.

The accidental $U(1)_{\mathrm{PQ}}$ charges are shown in the last row of Table V. Since the mixed anomaly with $S U(3)_{C}$ is nonvanishing, the strong $C P$ problem is solved if the $U(1)_{\mathrm{PQ}}$ symmetry is a sufficiently precise symmetry. The explicit PQ breaking superpotential is given by

$$
W_{\mathrm{PQ}}=\frac{w_{0}}{\Lambda^{4}} \mathcal{H}^{2} P^{4}+\frac{w_{0}}{\Lambda^{10}} P^{9} \bar{P}^{3}+\cdots .
$$

The full list of PQ breaking operators in the superpotential and Kähler potential are shown in Table VIII of Appendix B. We see that the first term in Eq. (60) and $K_{\mathrm{QQ}} \supset \mathcal{H}^{2} P^{4}$ give $\Delta \theta \sim 10^{-14}$ which are sufficiently small. Therefore, the PQ symmetry is high quality and the condition (2) is satisfied.

The leading RPV operators and linear terms in the vectorlike fields are given by

$$
\begin{aligned}
\Delta W \supset & \frac{\bar{P}}{\Lambda^{2}} Q Q S^{c} Q^{c}+\frac{w_{0} \bar{P}}{\Lambda^{2}} Q \overline{\mathcal{H}} S^{c} \\
& +\frac{P \bar{P}^{2}}{\Lambda^{2}}\left(\bar{S}^{c} Q^{c}+\frac{1}{\Lambda} Q \mathcal{H} S^{c}+\frac{1}{\Lambda} \Psi \overline{\mathcal{H}} S^{c}\right) \\
& +\frac{w_{0}}{\Lambda}\left(Q^{c} \Sigma Q^{c}+Q \bar{\Sigma} \Psi\right)+\cdots,
\end{aligned}
$$


where $Q^{c}$ includes $\Psi^{c}$ as the fourth family. The operators in the first line induce RPV effects without baryon number violation. The first two terms and the first term in the parenthesis are the dominant source for the lepton number violation, and the others are subdominant. The first term in the parenthesis induces the bilinear RPV operator $H_{u} \ell$ after integrating out the right-handed neutrino $\nu^{c}$. Its mass parameter is $\mathcal{O}\left(f_{\mathrm{PQ}}^{3} / v_{\mathrm{PS}} \Lambda\right)$, while that for $Q \overline{\mathcal{H}}$ from the second term is $\mathcal{O}\left(w_{0} f_{\mathrm{PQ}} v_{\mathrm{PS}} / \Lambda^{2}\right)$. The bilinear RPV from the second term in the parenthesis is smaller than these contributions. The last term would induce the bilinear RPV by the mixing of $Q$ and $\Psi$, but it is extremely small, unlike the mixing of $Q^{c}$ and $\Psi^{c}$, because the mass parameter for $Q \bar{\Psi}$ is $\mathcal{O}\left(10^{-22} \mathrm{GeV}\right)$. Note that all of these terms conserve the PQ symmetry, so that these interactions do not explicitly depend on the axion superfield. The operators relevant to masses, RPV and proton decay are listed in Table IX. We see that the other mass terms are at most $\mathcal{O}\left(10^{-13} \mathrm{GeV}\right)$ and are negligible. Therefore, the MSSM particles, $\overline{\mathcal{H}}$ and $(\sigma, \bar{\sigma})$ are lighter than the SUSY breaking scale $\mathcal{O}\left(w_{0}\right)$, while all the vectorlike fields, $\Psi, \bar{\Psi}$, have $\mathcal{O}\left(f_{\mathrm{PQ}}\right)$ masses. The gauge coupling unification holds as discussed in the previous section and the condition (5) is satisfied.

Except for the operator $Q^{c} \bar{\Psi}^{c}$, the mass terms which contribute to $\mu_{T}$ and $\mu_{\bar{T}}$ in Eq. (36) are smaller than $10^{-21} \mathrm{GeV}$, see Table IX. These are too small to affect the proton stability. The mixing via $Q^{c} \bar{\Psi}^{c}$ is sizable, but this can be rotated away by redefining $Q^{c}$ and $\Psi^{c}$ without introducing new effects because $\Psi^{c}$ has the same charges as $Q^{c}$. As is explicitly shown in Table IX, the coupling constants for the operators linear in the vectorlike fields, defined in Eq. (36), are $\lambda_{T} \lesssim 10^{-18}$ and $\kappa_{T} \lesssim 10^{-31} \mathrm{GeV}^{-1}$, except for those in Eq. (61). Hence, only the operators in Eq. (61) could induce fast proton decay. The vectorlike pair, $(\Psi, \bar{\Psi})$, is integrated out at $\mathcal{O}\left(f_{\mathrm{PQ}}\right)$ before integrating out $(\sigma, \bar{\sigma})$ whose mass is $\mathcal{O}\left(w_{0}\right)$. Since $\bar{\Psi}$ and $\bar{\Psi}^{c}$ are absent in Eq. (61), there will be no sizable baryon number violation in the superpotential. In addition, there cannot be a sizable dimension-6 operator in the Kähler potential, since the Yukawa coupling involving the vectorlike triplets is at most $\mathcal{O}\left(10^{-13}\right)$ from the first term in the second line of Eq. (61). Thus, R-parity is broken by the lepton number violating operator, while baryon number is still a precise symmetry such that the proton is stable.

If the neutralino is the LSP, the lifetime of the LSP is as short as $10^{-10} \mathrm{~s}$, see Eqs. (31) and (32), due to the Higgsino-neutrino mixing by the second term in Eq. (61). Thus the neutralino LSP is unstable and will decay before BBN.

The axino LSP may be sufficiently long-lived even with RPV. The axion superfield $A$ is defined as

$$
P \sim f_{\mathrm{PQ}} e^{-4 A / f_{\mathrm{PQ}}}, \quad \bar{P} \sim f_{\mathrm{PQ}} e^{2 A / f_{\mathrm{PQ}}} .
$$

In the RPV model, the axino will mix with Higgsinos in the Kähler potential [62],

$$
\begin{aligned}
K & \supset e^{\left(A+A^{\dagger}\right) / f_{\mathrm{PQ}}} \mathcal{H}^{\dagger} \mathcal{H}+e^{-\left(A+A^{\dagger}\right) / f_{\mathrm{PQ}}} \overline{\mathcal{H}}^{\dagger} \overline{\mathcal{H}} \\
& \supset \frac{v_{H}}{f_{\mathrm{PQ}}}\left(H_{d}^{\dagger} A+\bar{H}_{u}^{\dagger} A\right),
\end{aligned}
$$

where \pm 1 in the exponents are the PQ charges of the Higgs bidoublets. Note that this mixing with the MSSM fields is absent in the minimal model, since those are neutral under the PQ symmetry. Together with the bilinear RPV term, the axino-neutrino mixing arises, so that the axino will decay via $\tilde{a} \rightarrow \nu \ell \ell^{\dagger}$. The lifetime is estimated as

$$
\begin{aligned}
\tau_{\tilde{a}} \sim & \frac{1536 \pi^{3}}{g_{\nu Z}^{4}} \frac{v_{H}^{2} v_{\mathrm{PS}}^{2} \Lambda^{2}}{f_{\mathrm{PQ}}^{4} m_{\tilde{a}}^{3}} \min \left(1, \frac{f_{\mathrm{PQ}}^{4} \Lambda^{2}}{w_{0}^{2} v_{\mathrm{PS}}^{4}}\right) \\
\sim & 10^{27} \text { years } \times\left(\frac{0.1}{g_{\nu Z}}\right)^{4}\left(\frac{1 \mathrm{keV}}{m_{\tilde{a}}}\right)^{3}\left(\frac{\Lambda}{10^{18} \mathrm{GeV}}\right)^{2} \\
& \times\left(\frac{v_{\mathrm{PS}}}{10^{16} \mathrm{GeV}}\right)^{2}\left(\frac{10^{10} \mathrm{GeV}}{f_{\mathrm{PQ}}}\right)^{4},
\end{aligned}
$$

where the value of min, which depends on whether the second or third term in Eq. (61) dominates the axinoneutrino mixing, is taken to be 1 in the second line. The axino-neutrino mixing induced by the PQ breaking interactions, Eq. (60), are much more suppressed than that induced by the RPV, but PQ conserving effects. In particular, the axion-axino-neutrino Yukawa coupling induced by the PQ breaking interactions is highly suppressed. Therefore, the axino lifetime, assuming a mass of order $1 \mathrm{keV}$, will be much longer than the age of the universe, although it is much shorter than that in the minimal model.

Altogether, this model has the high-quality axion and the proton is stable, and thus satisfies all of the conditions (1)(5). If the neutralino is the LSP, the overproduction problem is solved because it decays before BBN. The axion would be the dominant source for the DM. If the axino is the LSP, the overproduction problem is solved by sufficiently light axino mass as in the minimal model. The axino will be a metastable DM particle in addition to the axion DM.

\section{DISCUSSIONS}

In this paper, we proposed supersymmetric Pati-Salam models with the anomaly-free discrete symmetry $\mathbb{Z}_{4}^{R} \times \mathbb{Z}_{N}$. The anomalous $U(1)_{\mathrm{PQ}}$ symmetry, as well as, R-parity arise as accidental symmetries if any of the PQ fields $P$ and $\bar{P}$ have odd R-charge. We discussed two special models. In the minimal model, without $\overline{\mathcal{H}}, \bar{\Sigma}, \bar{P}$, the anomaly conditions require that the MSSM particles do not carry $\mathbb{Z}_{N}$ charges, so that the R-parity is respected very accurately and the LSP is sufficiently stable to be the DM. In the nonminimal cases, we found an example which violates 
R-parity such that the neutralino LSP will decay before $\mathrm{BBN}$, while the accidental $U(1)_{\mathrm{PQ}}$ symmetry is so accurate that the strong $C P$ problem is solved. An interesting feature of the RPV case is that the exotic vectorlike triplets $(\sigma, \bar{\sigma})$ and bidoublet $\overline{\mathcal{H}}$ are predicted to have SUSY breaking scale masses. Since the vectorlike triplets may decay through the Yukawa couplings which are also induced by the SUSY breaking effects, the SUSY breaking scale is predicted to be larger than $\mathcal{O}(100 \mathrm{TeV})$.

With the discrete symmetries, there are self-couplings of the PQ field $P$ at very high-order. It was recently proposed that the baryon asymmetry can be produced through the motion of a PQ field when kicked by an A-term of the selfcoupling $P^{n}$, so-called, lepto-axiogenesis $[64,65]$. Our models may provide concrete examples which can accommodate the lepto-axiogenesis scenario. In particular, the RPV example will make it easier to explain the relic density of the DM. A more detailed analysis of lepto-axiogenesis and the phenomenological discussions about the DM and leptogenesis will be the subject of future work.

\section{ACKNOWLEDGMENTS}

The work of J.K and S.R is supported in part by the Department of Energy (DOE) under Award No. DESC0011726. This work of J. K. is supported in part by the Grant-in-Aid for Scientific Research from the Ministry of Education, Science, Sports and Culture (MEXT), Japan No. 18 K13534.

\section{APPENDIX A: HIGGS SECTOR IN THE NONMINIMAL MODEL}

We shall study the Higgs potential with the additional bidoublet $\overline{\mathcal{H}}$. We write the bidoublets by

$$
\mathcal{H}=\left(\begin{array}{l}
H_{1} \\
H_{2}
\end{array}\right), \quad \overline{\mathcal{H}}=\left(\begin{array}{c}
H_{3} \\
H_{4}
\end{array}\right),
$$

where $H_{i}, i=1,2,3,4$, are $S U(2)_{L}$ doublets. The superpotential is given by

$$
W_{\mathrm{PS}}=\frac{1}{2} \mu \mathcal{H}^{2}+w_{0} \mathcal{H} \overline{\mathcal{H}} \rightarrow W_{H}=\mu_{k l} H_{k} H_{l},
$$

where $k=1,3$ and $l=2,4$. In this section, repeated indices are summed over. With the PS symmetry, $\mu_{12}=\mu$, $\mu_{14}=-\mu_{32}=w_{0}$ and $\mu_{34}=0$, but this relation will not hold after the PS breaking. The $S U(2)_{L}$ doublets are contracted by $i \sigma_{2}$. We shall study the Higgs potential in the nonminimal model given by

$$
V_{H}=V_{\text {soft }}+V_{F}+V_{D},
$$

with

$$
\begin{gathered}
V_{\text {soft }}=\sum_{i=1}^{4} m_{H_{i}}^{2}\left|H_{i}\right|^{2}+\left(b_{k l} H_{k} H_{l}+\text { H.c. }\right), \\
V_{F}=\sum_{k=1,3}\left|\mu_{k l} H_{l}\right|^{2}+\sum_{l=2,4}\left|\mu_{k l} H_{k}\right|^{2} \\
V_{D}=\frac{g_{1}^{2}}{8}\left(\left|H_{1}\right|^{2}-\left|H_{2}\right|^{2}+\left|H_{3}\right|^{2}-\left|H_{4}\right|^{2}\right)^{2} \\
+\frac{g_{2}^{2}}{2}\left(\sum_{i=1}^{4} H_{i}^{*} T_{L}^{a} H_{i}\right)^{2},
\end{gathered}
$$

where $T_{L}^{a}, a=1,2,3$, is generators of $S U(2)_{L}$. We first diagonalize the mass terms by redefining the Higgs fields,

$$
\left(\begin{array}{c}
H_{1} \\
H_{3}
\end{array}\right)=: R_{u}\left(\begin{array}{c}
H_{u} \\
\bar{H}_{d}
\end{array}\right), \quad\left(\begin{array}{c}
H_{2} \\
H_{4}
\end{array}\right)=: R_{d}\left(\begin{array}{c}
H_{d} \\
\bar{H}_{u}
\end{array}\right)
$$

where the rotation matrices $R_{u}, R_{d}$ diagonalize the Higgs mass squared matrices,

$$
\begin{gathered}
R_{u}^{\dagger}\left(\begin{array}{cc}
m_{H_{1}}^{2}+\mu_{1 l}^{*} \mu_{1 l} & \mu_{1 l}^{*} \mu_{3 l} \\
\mu_{3 l}^{*} \mu_{1 l} & m_{H_{3}}^{2}+\mu_{3 l}^{*} \mu_{3 l}
\end{array}\right) R_{u}=: \operatorname{diag}\left(m_{H_{u}}^{2}, \bar{m}_{H_{d}}^{2}\right), \\
R_{d}^{\dagger}\left(\begin{array}{cc}
m_{H_{2}}^{2}+\mu_{k 2}^{*} \mu_{k 2} & \mu_{k 2}^{*} \mu_{k 4} \\
\mu_{k 4}^{*} \mu_{k 2} & m_{H_{4}}^{2}+\mu_{k 4}^{*} \mu_{k 4}
\end{array}\right) R_{d}=: \operatorname{diag}\left(m_{H_{d}}^{2}, \bar{m}_{H_{u}}^{2}\right),
\end{gathered}
$$

where $k=1,3$ and $l=2,4$ are summed over. The D-term potential is invariant under this redefinition, so that it is formally replaced by $\left(H_{1}, H_{2}, H_{3}, H_{4}\right) \rightarrow\left(H_{u}, H_{d}, \bar{H}_{d}, \bar{H}_{u}\right)$. The $b$-terms are rotated as

$$
\begin{aligned}
& \left(\begin{array}{ll}
H_{1} & H_{3}
\end{array}\right)\left(\begin{array}{ll}
b_{12} & b_{14} \\
b_{32} & b_{34}
\end{array}\right)\left(\begin{array}{c}
H_{2} \\
H_{4}
\end{array}\right) \\
& =\left(\begin{array}{ll}
H_{u} & \bar{H}_{d}
\end{array}\right)\left(\begin{array}{ll}
b_{h} & b_{u} \\
b_{d} & \bar{b}_{h}
\end{array}\right)\left(\begin{array}{c}
H_{d} \\
\bar{H}_{u}
\end{array}\right),
\end{aligned}
$$

where

$$
\left(\begin{array}{ll}
b_{h} & b_{u} \\
b_{d} & \bar{b}_{h}
\end{array}\right):=R_{u}^{T}\left(\begin{array}{ll}
b_{12} & b_{14} \\
b_{32} & b_{34}
\end{array}\right) R_{d}
$$

The Higgs potential after the rotation is

$$
\begin{aligned}
V_{H}= & m_{H_{u}}^{2}\left|H_{u}\right|^{2}+m_{H_{d}}^{2}\left|H_{d}\right|^{2}+\bar{m}_{H_{d}}^{2}\left|\bar{H}_{d}\right|^{2}+\bar{m}_{H_{u}}^{2}\left|\bar{H}_{u}\right|^{2} \\
& +\left(b_{h} H_{u} H_{d}+b_{u} H_{u} \bar{H}_{u}+b_{d} \bar{H}_{d} H_{d}+\bar{b}_{h} \bar{H}_{d} \bar{H}_{u}+\right.\text { H.c.) } \\
& +V_{D} .
\end{aligned}
$$

The first derivatives of the neutral Higgs potential are given by 


$$
\begin{gathered}
\frac{\partial V_{H}}{\partial H_{u}^{0 *}}=m_{H_{u}}^{2} H_{u}^{0}+\lambda_{H} \Omega H_{u}^{0}-b_{h} H_{d}^{0 *}-b_{u} \bar{H}_{u}^{0 *}, \\
\frac{\partial V_{H}}{\partial H_{d}^{0 *}}=m_{H_{d}}^{2} H_{d}^{0}-\lambda_{H} \Omega H_{d}^{0}-b_{h} H_{u}^{0 *}-b_{d} \bar{H}_{d}^{0 *}, \\
\frac{\partial V_{H}}{\partial \bar{H}_{d}^{0 *}}=\bar{m}_{H_{d}}^{2} \bar{H}_{d}^{0}+\lambda_{H} \Omega \bar{H}_{d}^{0}-\bar{b}_{h} \bar{H}_{u}^{0 *}-b_{d} H_{d}^{0 *}, \\
\frac{\partial V_{H}}{\partial \bar{H}_{u}^{0 *}}=\bar{m}_{H_{u}}^{2} \bar{H}_{u}^{0}-\lambda_{H} \Omega \bar{H}_{u}^{0}-\bar{b}_{h} \bar{H}_{d}^{0 *}-b_{u} H_{u}^{0 *},
\end{gathered}
$$

where

$$
\begin{aligned}
\lambda_{H} & :=\frac{g_{1}^{2}+g_{2}^{2}}{4} \\
\Omega & :=\left|H_{u}^{0}\right|^{2}-\left|H_{d}^{0}\right|^{2}+\left|\bar{H}_{d}^{0}\right|^{2}-\left|\bar{H}_{u}^{0}\right|^{2} .
\end{aligned}
$$

The Higgs fields with superscript 0 are the neutral component of the Higgs doublets.

We define the VEVs and Higgs scalars in the doublets as

$$
\begin{aligned}
& H_{u}=\left(\begin{array}{c}
0 \\
v_{u}
\end{array}\right)+\frac{1}{\sqrt{2}}\left(\begin{array}{c}
\sqrt{2} H_{u}^{+} \\
h_{u}+i a_{u}
\end{array}\right), \\
& H_{d}=\left(\begin{array}{c}
v_{d} \\
0
\end{array}\right)+\frac{1}{\sqrt{2}}\left(\begin{array}{c}
h_{d}+i a_{d} \\
\sqrt{2} H_{d}^{-}
\end{array}\right), \\
& \bar{H}_{d}=\left(\begin{array}{c}
0 \\
\bar{v}_{d}
\end{array}\right)+\frac{1}{\sqrt{2}}\left(\begin{array}{c}
\sqrt{2} \bar{H}_{d}^{+} \\
\bar{h}_{d}+i \bar{a}_{d}
\end{array}\right), \\
& \bar{H}_{u}=\left(\begin{array}{c}
\bar{v}_{u} \\
0
\end{array}\right)+\frac{1}{\sqrt{2}}\left(\begin{array}{c}
\bar{h}_{u}+i \bar{a}_{u} \\
\sqrt{2} \bar{H}_{u}^{-}
\end{array}\right) .
\end{aligned}
$$

Elements of the $C P$-even mass matrix are given by

$$
\begin{aligned}
& {\left[\mathcal{M}_{S}^{2}\right]_{h_{u} h_{u}}=2 \lambda_{H} v_{u}^{2}+\left(b_{h} v_{d}+b_{u} \bar{v}_{u}\right) / v_{u},} \\
& {\left[\mathcal{M}_{S}^{2}\right]_{h_{u} h_{d}}=-2 \lambda_{H} v_{u} v_{d}-b_{h},} \\
& {\left[\mathcal{M}_{S}^{2}\right]_{h_{u} \bar{h}_{d}}=2 \lambda_{H} v_{u} \bar{v}_{d}, \quad\left[\mathcal{M}_{S}^{2}\right]_{h_{u} \bar{h}_{u}}=-2 \lambda_{H} v_{u} \bar{v}_{u}-b_{u},} \\
& {\left[\mathcal{M}_{S}^{2}\right]_{h_{d} h_{d}}=2 \lambda_{H} v_{d}^{2}+\left(b_{h} v_{u}+b_{d} \bar{v}_{d}\right) / v_{d},} \\
& {\left[\mathcal{M}_{S}^{2}\right]_{h_{d} \bar{h}_{d}}=-2 \lambda_{H} v_{d} \bar{v}_{d}-b_{d},} \\
& {\left[\mathcal{M}_{S}^{2}\right]_{h_{d} \bar{h}_{u}}=2 \lambda_{H} v_{d} \bar{v}_{u},} \\
& {\left[\mathcal{M}_{S}^{2}\right]_{\bar{h}_{d} \bar{h}_{d}}=2 \lambda_{H} \bar{v}_{d}^{2}+\left(b_{d} v_{d}+\bar{b}_{h} \bar{v}_{u}\right) / \bar{v}_{d},} \\
& {\left[\mathcal{M}_{S}^{2}\right]_{\bar{h}_{d} \bar{h}_{u}}=-2 \lambda \bar{v}_{u} \bar{v}_{d}-\bar{b}_{h},} \\
& {\left[\mathcal{M}_{S}^{2}\right]_{\bar{h}_{u} \bar{h}_{u}}=2 \lambda_{h} \bar{v}_{u}^{2}+\left(b_{u} v_{u}+\bar{b}_{u} \bar{v}_{d}\right) / \bar{v}_{u},}
\end{aligned}
$$

and those of the $C P$-odd mass matrix are given by

$$
\begin{aligned}
& {\left[\mathcal{M}_{P}^{2}\right]_{a_{u} a_{u}}=\left(b_{h} v_{d}+b_{u} \bar{v}_{u}\right) / v_{u}, \quad\left[\mathcal{M}_{P}^{2}\right]_{a_{u} a_{d}}=b_{h},} \\
& {\left[\mathcal{M}_{P}^{2}\right]_{a_{u} \bar{a}_{d}}=0, \quad\left[\mathcal{M}_{P}^{2}\right]_{a_{u} \bar{a}_{u}}=b_{u},} \\
& {\left[\mathcal{M}_{P}^{2}\right]_{a_{d} a_{d}}=\left(b_{h} v_{u}+b_{d} \bar{v}_{d}\right) / v_{d}, \quad\left[\mathcal{M}_{P}^{2}\right]_{a_{d} \bar{a}_{d}}=b_{d},} \\
& {\left[\mathcal{M}_{P}^{2}\right]_{a_{d} \bar{a}_{u}}=0,} \\
& {\left[\mathcal{M}_{P}^{2}\right]_{\bar{a}_{d} \bar{a}_{d}}=\left(b_{d} v_{d}+\bar{b}_{h} \bar{v}_{u}\right) / \bar{v}_{d}, \quad\left[\mathcal{M}_{P}^{2}\right]_{\bar{a}_{d} \bar{a}_{u}}=\bar{b}_{h},} \\
& {\left[\mathcal{M}_{P}^{2}\right]_{\bar{a}_{u} \bar{a}_{u}}=\left(b_{u} v_{u}+\bar{b}_{h} \bar{v}_{d}\right) / \bar{v}_{u} .}
\end{aligned}
$$

Here, the soft masses are replaced by using the minimization conditions.

Let us consider the realistic EW vacuum, $v_{u}, v_{d} \gg \bar{v}_{u}$, $\bar{v}_{d}$. We define

$v_{u}:=v_{h} s_{\beta}, \quad v_{d}:=v_{h} c_{\beta}, \quad \bar{v}_{d}:=\bar{v}_{h} \bar{c}_{\beta}$,

$\bar{v}_{u}:=\bar{v}_{h} \bar{s}_{\beta}, \quad t_{\beta}:=\frac{s_{\beta}}{c_{\beta}}, \quad \bar{t}_{\beta}:=\frac{\bar{s}_{\beta}}{\bar{c}_{\beta}}$.

Assuming $\bar{m}_{H_{d}}^{2}, \bar{m}_{H_{u}}^{2} \gg v_{h}^{2}, \bar{v}_{h}^{2}$, the minimization conditions for $\bar{H}_{d}, \bar{H}_{u}$ become

$\frac{\bar{v}_{h}}{v_{h}} \sim \frac{b_{d} c_{\beta} \bar{c}_{\beta}-b_{u} s_{\beta} \bar{s}_{\beta}}{\bar{m}_{H_{d}}^{2} \bar{c}_{\beta}^{2}-\bar{m}_{H_{u}}^{2} \bar{s}_{\beta}^{2}}, \quad \bar{t}_{\beta} \sim \frac{b_{d} \bar{b}_{h}+b_{u} \bar{m}_{H_{d}}^{2} t_{\beta}}{b_{d} \bar{m}_{H_{u}}^{2}+b_{u} \bar{b}_{h} t_{\beta}}$.

Thus, $\bar{m}_{H_{u}}^{2}, \bar{m}_{H_{d}}^{2} \gg b_{u}, \quad b_{d}$ is required to be $\bar{v}_{h} \ll v_{h}$. Neglecting $\mathcal{O}\left(\bar{v}_{h}^{2}\right)$, the minimization conditions for $H_{u}$, $H_{d}$ are given by

$$
\begin{gathered}
m_{H_{u}}^{2}-\lambda_{H} v_{h}^{2} c_{2 \beta}-b_{h} / t_{\beta}=b_{u} \frac{\bar{v}_{h} \bar{s}_{\beta}}{v_{h} s_{\beta}}, \\
m_{H_{d}}^{2}+\lambda_{H} v_{h}^{2} c_{2 \beta}-b_{h} t_{\beta}=b_{d} \frac{\bar{v}_{h} \bar{c}_{\beta}}{v_{h} c_{\beta}} .
\end{gathered}
$$

The Higgs VEV $v_{h}$ and vacuum angle $\beta$ obey

$$
\lambda_{H} v_{h}^{2}=\frac{\tilde{m}_{H_{d}}^{2}-\tilde{m}_{H_{u}}^{2} t_{\beta}^{2}}{t_{\beta}^{2}-1}, \quad s_{2 \beta}=\frac{2 b_{h}}{\tilde{m}_{H_{u}}^{2}+\tilde{m}_{H_{d}}^{2}},
$$

where

$$
\tilde{m}_{H_{u}}^{2}:=m_{H_{u}}^{2}-b_{u} \frac{\bar{v}_{h} \bar{s}_{\beta}}{v_{h} s_{\beta}}, \quad \tilde{m}_{H_{d}}^{2}:=m_{H_{d}}^{2}-b_{d} \frac{\bar{v}_{h} \bar{c}_{\beta}}{v_{h} c_{\beta}} .
$$

Note that $m_{H_{u}}^{2}, m_{H_{d}}^{2}$ contain the SUSY contributions from the $\mu$-parameters. The SUSY breaking parameters should be fine-tuned to realize $\lambda_{H} v_{h}^{2} \sim m_{Z}^{2}=91.2 \mathrm{GeV}$. In the RPV model, $\mu \sim 100 \mathrm{GeV}$ and $w_{0} \sim 10^{5} \mathrm{GeV}$, so the mixing in $R_{u}, R_{d}$ are suppressed by $\sim w_{0} \mu / m_{H_{3,4}}^{2}$. Hence, the mass parameters $m_{H_{u}}^{2}, m_{H_{d}}^{2}$ which directly relate to the EW scale, are approximately given by $m_{H_{1,2}}^{2}+\left|w_{0}\right|^{2}+|\mu|^{2}$ even if $m_{H_{3,4}}^{2} \gg b_{u, d}$ for $\bar{v}_{h} / v_{h} \ll 1$. Therefore, the degree 
of fine-tuning for the EW symmetry breaking is about $\mathcal{O}\left(w_{0}^{2} / m_{Z}^{2}\right) \sim \mathcal{O}\left(10^{6}\right)$. We also remark that the relation $m_{H_{1,2}}^{2} \ll m_{H_{3,4}}^{2}$ would be naturally realized as a result of renormalization group running because only $\mathcal{H}$ has the sizable Yukawa couplings with the MSSM quarks.

Before closing, let us estimate the heavy Higgs boson mass spectrum. Neglecting $\mathcal{O}\left(v_{h}^{2}, b_{u, d} \bar{v}_{h} / v_{h}\right)$, the $C P$-even and $C P$-odd mass matrices are given by

$\mathcal{M}_{S}^{2} \sim \mathcal{M}_{P}^{2} \sim\left(\begin{array}{cccc}b_{h} / t_{\beta} & b_{h} & 0 & b_{u} \\ b_{h} & b_{h} t_{\beta} & b_{d} & 0 \\ 0 & b_{d} & b_{d} v_{d} / \bar{v}_{d} & \bar{b}_{h} \\ b_{u} & 0 & \bar{b}_{h} & b_{u} v_{u} / \bar{v}_{u}\end{array}\right)$.

Since $\mathcal{M}_{P}^{2}=\mathcal{M}_{S}^{2}+\mathcal{O}\left(v_{h}^{2}\right)$ is rank-3, one eigenvalue is zero. The zero eigenstate in the $C P$-even Higgs boson corresponds to the SM Higgs boson whose mass comes from the $\mathcal{O}\left(v_{h}^{2}\right)$ correction, and that in the $C P$-odd Higgs boson corresponds to the NG boson absorbed by a $Z$-boson. The other three states have masses of $b_{h}$, $b_{d} v_{d} / \bar{v}_{d} \sim \bar{m}_{H_{d}}^{2}$ and $b_{u} v_{u} / \bar{v}_{u} \sim \bar{m}_{H_{u}}^{2}$.

\section{APPENDIX B: DETAILS OF THE EXAMPLE MODELS}

In this Appendix, we shall show possible operators which can induce the shift in the $\theta$ angle, masses and proton decay. Their sizes are calculated with

$$
\Lambda=10^{18} \mathrm{GeV}, \quad v_{\mathrm{PS}}=10^{16} \mathrm{GeV}, \quad f_{\mathrm{PQ}}=10^{10} \mathrm{GeV},
$$

$w_{0}=10^{5} \mathrm{GeV}, \quad v_{H}=10^{2} \mathrm{GeV}$,

as reference values.

Table VI lists the PQ breaking operators and their effects to $\Delta \theta$ in the minimal model. The tree-level PQ breaking effects are shown in the columns of $\mathcal{O}=\mathbf{1}$. The others are

TABLE VI. Sizes of $\Delta \theta$ in the minimal model.

\begin{tabular}{lcc}
\hline \hline Operator $\mathcal{O} \in W_{\mathrm{PQ}}$ & Coupling & $\log _{10} \Delta \theta$ \\
\hline $\mathbf{1}$ & $P^{10}$ & -17 \\
$\mathcal{H}^{2}$ & $P^{10}$ & -112 \\
$Q^{c} \bar{S}^{c}$ & $P^{5}$ & -32 \\
$Q^{c} \bar{\Psi}^{c}$ & $P^{11} w_{0}$ & -154 \\
$\bar{S}^{c} S^{c}$ & $P^{10}$ & -112 \\
$\bar{S}^{c} \Psi^{c}$ & $P^{5}$ & -32 \\
$S^{c} \bar{\Psi}^{c}$ & $P^{6}$ & -48 \\
$X^{2}$ & $P^{10}$ & -112 \\
$\Sigma^{2}$ & $P^{10}$ & -112 \\
$Q \bar{\Psi}$ & $P^{11} w_{0}$ & -154 \\
$\Psi \bar{\Psi}$ & $P^{11} w_{0}$ & -154 \\
$\Psi c \bar{\Psi}^{c}$ & $P^{11} w_{0}$ & -154 \\
\hline
\end{tabular}

(Table continued)

TABLE VI. (Continued)

\begin{tabular}{lcc}
\hline \hline Operator $\mathcal{O} \in W_{\mathrm{PQ}}$ & Coupling & $\log _{10} \Delta \theta$ \\
\hline $\mathcal{H} Q S^{c}$ & $P^{5}$ & -36 \\
$\mathcal{H} S^{c} \Psi$ & $P^{5}$ & -36 \\
$Q^{c} S^{c} \Sigma$ & $P^{5} w_{0}$ & -62 \\
$S^{c} \Sigma \Psi^{c}$ & $P^{5} w_{0}$ & -62 \\
$\mathcal{H} \bar{S}^{c} \bar{\Psi}$ & $P^{6}$ & -52 \\
$\bar{S}^{c} \Sigma \bar{\Psi}^{c}$ & $P^{6} w_{0}$ & -78 \\
\hline
\end{tabular}

\begin{tabular}{lcr}
\hline \hline Operator $\mathcal{O} \in K_{\mathrm{PQ}}$ & Coupling & $\log _{10} \Delta \theta$ \\
\hline $\mathbf{1}$ & $P^{10} w_{0}$ & -43 \\
$\mathcal{H}^{2}$ & $P^{10} w_{0}$ & -164 \\
$Q^{c} Q^{c \dagger}$ & $P^{10} w_{0}$ & -164 \\
$Q^{c} \bar{S}^{c}$ & $P^{5} w_{0}$ & -84 \\
$Q^{c} \bar{\Psi}^{c}$ & $P^{11}$ & -154 \\
$Q^{c} \Psi^{c \dagger}$ & $P^{10} w_{0}$ & -164 \\
$Q^{c \dagger} S^{c}$ & $P^{5}$ & -58 \\
$Q^{c \dagger} \Psi^{c}$ & $P^{10} w_{0}$ & -164 \\
$Q^{c \dagger} \bar{\Psi}^{c \dagger}$ & $P^{9}$ & -122 \\
$Q Q^{\dagger}$ & $P^{10} w_{0}$ & -164 \\
$Q^{\dagger}$ & $P^{11}$ & -154 \\
\hline
\end{tabular}

\begin{tabular}{|c|c|c|}
\hline Operator $\mathcal{O} \in K_{\mathrm{PQ}}$ & Coupling & $\log _{10} \Delta \theta$ \\
\hline$Q \Psi^{\dagger}$ & $P^{10} w_{0}$ & -164 \\
\hline$Q^{\dagger} \Psi$ & $P^{10} w_{0}$ & -164 \\
\hline$Q^{\dagger} \bar{\Psi}^{\dagger}$ & $P^{9}$ & -122 \\
\hline $\bar{S}^{c} S^{c}$ & $P^{10} w_{0}$ & -164 \\
\hline $\bar{S}^{c} \Psi^{c}$ & $P^{5} w_{0}$ & -84 \\
\hline $\bar{S}^{c} \bar{\Psi}^{c \dagger}$ & $P^{4}$ & -42 \\
\hline$S^{c} \bar{\Psi}^{c}$ & $P^{6} w_{0}$ & -100 \\
\hline$S^{c} \Psi^{c^{\dagger}}$ & $P^{5}$ & -58 \\
\hline$X^{2}$ & $P^{10} w_{0}$ & -164 \\
\hline$\Sigma^{2}$ & $P^{10} w_{0}$ & -164 \\
\hline$\Psi \bar{\Psi}$ & $P^{11}$ & -154 \\
\hline$\Psi \Psi^{\dagger}$ & $P^{10} w_{0}$ & -164 \\
\hline $\bar{\Psi} \bar{\Psi} \dagger$ & $P^{10} w_{0}$ & -164 \\
\hline $\bar{\Psi} \Psi^{\dagger}$ & $P^{9}$ & -122 \\
\hline$\Psi^{c} \bar{\Psi}^{c}$ & $P^{11}$ & -154 \\
\hline$\Psi^{c} \Psi^{c \dagger}$ & $P^{10} w_{0}$ & -164 \\
\hline $\bar{\Psi}^{c} \bar{\Psi}^{c \dagger}$ & $P^{10} w_{0}$ & -164 \\
\hline $\bar{\Psi}^{c \dagger} \Psi^{c \dagger}$ & $P^{9}$ & -122 \\
\hline $\mathcal{H} Q S^{c}$ & $P^{5} w_{0}$ & -88 \\
\hline $\mathcal{H} S^{c} \Psi$ & $P^{5} w_{0}$ & -88 \\
\hline $\mathcal{H} S^{c} \overline{\boldsymbol{\Psi}}^{\dagger}$ & $P^{4}$ & -46 \\
\hline$Q^{c} S^{c} \Sigma$ & $P^{5}$ & -62 \\
\hline$S^{c} \Sigma \Psi^{c}$ & $P^{5}$ & -62 \\
\hline$S^{c} \Sigma \bar{\Psi}^{c \dagger}$ & $P^{4} w_{0}$ & -72 \\
\hline $\mathcal{H} Q^{\dagger} \bar{S}^{c}$ & $P^{5}$ & -62 \\
\hline $\mathcal{H} \bar{S}^{c} \bar{\Psi}$ & $P^{6} w_{0}$ & -104 \\
\hline $\mathcal{H} \bar{S}^{c} \Psi^{\dagger}$ & $P^{5}$ & -62 \\
\hline$Q^{c \dagger} \bar{S}^{c} \Sigma$ & $P^{5} w_{0}$ & -88 \\
\hline $\bar{S}^{c} \Sigma \bar{\Psi}^{c}$ & $P^{6}$ & -78 \\
\hline $\bar{S}^{c} \Sigma \Psi^{c \dagger}$ & $P^{5} w_{0}$ & -88 \\
\hline
\end{tabular}


operators which can affect to the $\theta$ angle through the 1-loop effects. We list all the possible quadratic operators in the superpotential and Kähler potential. The cubic operators linearly depending on $S^{c}$ or $\bar{S}^{c}$ become quadratic ones after the PS breaking. For example, we see

$$
W_{\mathrm{PQ}} \supset \frac{P^{5}}{\Lambda^{5}} \times \mathcal{H} Q S^{c} \sim \frac{f_{\mathrm{PQ}}^{5}}{\Lambda^{5}} v_{\mathrm{PS}} \ell H_{u}
$$

induces $\Delta \theta \sim 10^{-36}$ at 1-loop level. In the minimal model, only the tree-level PQ breaking in the superpotential induces $\Delta \theta>10^{-20}$.

TABLE VII. Sizes of masses (left) and coupling constants for dimension-4 and -5 operators which can be relevant to proton decay (right) in the minimal model.

\begin{tabular}{|c|c|c|}
\hline Operator $\mathcal{O}$ & Mass $m_{\mathcal{O}}$ & $\log _{10} m_{\mathcal{O}}$ \\
\hline $\mathcal{H}^{2}$ & $w_{0}$ & 5 \\
\hline$Q^{c} \bar{S}^{c}$ & $P^{5}$ & -22 \\
\hline$Q^{c} \bar{\Psi}^{c}$ & $P$ & 10 \\
\hline $\bar{S}^{c} S^{c}$ & $w_{0}$ & 5 \\
\hline $\bar{S}^{c} \Psi^{c}$ & $P^{5}$ & -22 \\
\hline$S^{c} \bar{\Psi}^{c}$ & $P^{6}$ & -30 \\
\hline$X^{2}$ & $w_{0}$ & 5 \\
\hline$\Sigma^{2}$ & $w_{0}$ & 5 \\
\hline$Q \bar{\Psi}$ & $P$ & 10 \\
\hline$\Psi \bar{\Psi}$ & $P$ & 10 \\
\hline$\Psi^{c} \bar{\Psi}^{c}$ & $P$ & 10 \\
\hline $\bar{S}^{c} S^{c} X$ & 1 & 16 \\
\hline$S^{c} S^{c} \Sigma$ & 1 & 16 \\
\hline $\bar{S}^{c} \bar{S}^{c} \Sigma$ & 1 & 16 \\
\hline $\mathcal{H} Q S^{c}$ & $P^{5}$ & -24 \\
\hline $\mathcal{H} S^{c} \Psi$ & $P^{5}$ & -24 \\
\hline$Q^{c} S^{c} \Sigma$ & $P^{5} w_{0}$ & -37 \\
\hline$S^{c} \Sigma \Psi^{c}$ & $P^{5} w_{0}$ & -37 \\
\hline $\mathcal{H} \bar{S}^{c} \bar{\Psi}$ & $P^{6}$ & -32 \\
\hline $\bar{S}^{c} \Sigma \bar{\Psi}^{c}$ & $P^{6} w_{0}$ & -45 \\
\hline Operator $\mathcal{O}$ & $\lambda_{\mathcal{O}}$ or $\kappa_{\mathcal{O}}$ & $\log _{10} \lambda_{\mathcal{O}}, \kappa_{\mathcal{O}}$ \\
\hline$\overline{Q^{2} Q^{c} S^{c}}$ & $P^{5} w_{0}$ & -55 \\
\hline$Q^{c 3} S^{c}$ & $P^{5} w_{0}$ & -55 \\
\hline$Q^{4}$ & $w_{0}$ & -31 \\
\hline$Q^{c 4}$ & $w_{0}$ & -31 \\
\hline$Q^{3} \Psi$ & $w_{0}$ & -31 \\
\hline$Q Q^{c 2} \Psi$ & $w_{0}$ & -31 \\
\hline$Q Q^{c} S^{c} \Psi$ & $P^{5} w_{0}$ & -55 \\
\hline$Q Q^{c} \bar{S}^{c} \bar{\Psi}$ & $P^{6} w_{0}$ & -63 \\
\hline$Q^{2} Q^{c} \Psi^{c}$ & $w_{0}$ & -31 \\
\hline$Q^{2} S^{c} \Psi^{c}$ & $P^{5} w_{0}$ & -55 \\
\hline$Q^{c 3} \Psi^{c}$ & $w_{0}$ & -31 \\
\hline $\mathbb{Q}^{c 2} S^{c} \Psi^{c}$ & $P^{5} w_{0}$ & -55 \\
\hline$Q^{2} \bar{S}^{c} \bar{\Psi}^{c}$ & $P^{6} w_{0}$ & -63 \\
\hline$Q^{c 2} \bar{S}^{c} \bar{\Psi}^{c}$ & $P^{6} w_{0}$ & -63 \\
\hline
\end{tabular}

Table VII shows the operators relevant to the masses (left) and proton decays (right) in the minimal model. Similarly to the PQ breaking at 1-loop level, we show all the quadratic operators and cubic operators which linearly depend on the PS breaking fields. The columns are highlighted if the mass term is larger than $10^{-9} \mathrm{GeV}$. The right table shows operators relevant to RPV and proton decay. The bilinear RPV operators are included in the left table for mass terms. We studied the dimension-4 RPV, dimension-5 proton decay operators and those which depend on the vectorlike fields linearly. If all the couplings of the operators are $\lambda_{\mathcal{O}}<10^{-17}$ for dimension-4 operators and $\kappa_{\mathcal{O}}<10^{-27} \mathrm{GeV}^{-1}$ for dimension-5 operators, which is true in the minimal model, the model satisfy the sufficient conditions to ensure the proton stability as discussed in Sec. IIE. Note that the coupling constants include the VEVs of the PS breaking fields.

Tables VIII and IX show the same as Tables VI and VII in the RPV model. In this analysis, we do not consider the nonzero VEV of $\overline{\mathcal{H}}^{2}$ because it has the same charge as $w_{0} P \bar{P}$ whose VEV is more than 3 orders of magnitude larger than that of $\left\langle\overline{\mathcal{H}}^{2}\right\rangle \ll v_{h}^{2}$. We see that the shift of the $\theta$ angle is

TABLE VIII. Sizes of $\Delta \theta$ in the RPV model.

\begin{tabular}{lcc}
\hline \hline Operator $\mathcal{O} \in W_{\mathrm{PQ}}$ & Coupling & $\log _{10} \Delta \theta$ \\
\hline $\mathbf{1}$ & $\mathcal{H}^{2} P^{4} w_{0}$ & -14 \\
$\mathcal{H}^{2}$ & $P^{8} w_{0}$ & -106 \\
$\overline{\mathcal{H}} \mathcal{H}$ & $\mathcal{H}^{2} P^{4} w_{0}$ & -106 \\
$\overline{\mathcal{H}}^{2}$ & $P^{8} \bar{P}^{2}$ & -112 \\
$Q^{c} \bar{S}^{c}$ & $P^{8} \bar{P} w_{0}$ & -122 \\
$Q^{c} \bar{\Psi}^{c}$ & $P^{7} w_{0}$ & -90 \\
$\bar{S}^{c} S^{c}$ & $\mathcal{H}^{2} P^{4} w_{0}$ & -106 \\
$\bar{S}^{c} \Psi^{c}$ & $P^{8} \bar{P} w_{0}$ & -122 \\
$S^{c} \bar{\Psi}^{c}$ & $P^{8} \bar{P}^{2} w_{0}$ & -138 \\
$X^{2}$ & $\mathcal{H}^{2} P^{4} w_{0}$ & -106 \\
$\Sigma^{2}$ & $\bar{P}^{6}$ & -48 \\
$\Sigma \bar{\Sigma}$ & $\mathcal{H}^{2} P^{4} w_{0}$ & -106 \\
$\bar{\Sigma}^{2}$ & $P^{4} \bar{P}^{2}$ & -48 \\
$Q \bar{\Psi}$ & $P^{9} \bar{P}^{2} w_{0}$ & -154 \\
$\Psi \bar{\Psi}$ & $\mathcal{H}^{2} \bar{P}^{5}$ & -96 \\
$\Psi^{c} \bar{\Psi}^{c}$ & $P^{7} w_{0}$ & -90 \\
$\mathcal{H} Q S^{c}$ & $P^{8} \bar{P}_{0}$ & -126 \\
$\mathcal{H} S^{c} \Psi$ & $P^{9} \bar{P}^{2}$ & -132 \\
$\overline{\mathcal{H}} Q S^{c}$ & $P^{7}$ & -68 \\
$\overline{\mathcal{H}} S^{c} \Psi$ & $P^{8} \bar{P}_{0}$ & -126 \\
$Q^{c} S^{c} \Sigma$ & $P^{8} \bar{P}$ & -100 \\
$Q^{c} S^{c} \bar{\Sigma}$ & $P^{3} w_{0}$ & -30 \\
$S^{c} \Sigma \Psi^{c}$ & $P^{8} \bar{P}$ & -100 \\
$S^{c} \bar{\Sigma} \Psi^{c}$ & $P^{3} w_{0}$ & -30 \\
$\mathcal{H} \bar{S}^{c} \bar{\Psi}$ & $P^{9} \bar{P} w_{0}$ & -142 \\
$\overline{\mathcal{H}} \bar{S}^{c} \bar{\Psi}$ & $P^{8}$ & -84 \\
\hline & & (Table continued \\
& &
\end{tabular}


TABLE VIII. (Continued)

\begin{tabular}{|c|c|c|}
\hline Operator $\mathcal{O} \in W_{\mathrm{PQ}}$ & Coupling & $\log _{10} \Delta \theta$ \\
\hline $\bar{S}^{c} \Sigma \bar{\Psi}^{c}$ & $P^{4} w_{0}$ & -46 \\
\hline $\bar{S}^{c} \bar{\Sigma} \bar{\Psi}^{c}$ & $P^{8} \bar{P}^{2}$ & -116 \\
\hline Operator $\mathcal{O} \in K_{\mathrm{PQ}}$ & Coupling & $\log _{10} \Delta \theta$ \\
\hline 1 & $\mathcal{H}^{2} P^{4}$ & -14 \\
\hline $\mathcal{H}^{2}$ & $P^{8}$ & -106 \\
\hline$\overline{\mathcal{H}} \mathcal{H}$ & $\mathcal{H}^{2} P^{4}$ & -106 \\
\hline$\overline{\mathcal{H}}^{2}$ & $P^{6} \bar{P}^{\dagger 2}$ & -106 \\
\hline$Q^{c} Q^{c \dagger}$ & $\mathcal{H}^{2} P^{4}$ & -106 \\
\hline$Q^{c} \overline{\bar{S}}^{c}$ & $\mathcal{H}^{2} P^{3} \bar{P}^{\dagger 2}$ & -122 \\
\hline$\hat{Q}^{c} \bar{\Psi}^{c}$ & $P^{7}$ & -90 \\
\hline$Q^{c} \Psi^{c \dagger}$ & $\mathcal{H}^{2} P^{4}$ & -106 \\
\hline$Q^{c \dagger} S^{c}$ & $P^{6} \bar{P}^{\dagger 3}$ & -122 \\
\hline$Q^{c \dagger} \Psi^{c}$ & $\mathcal{H}^{2} P^{4}$ & -106 \\
\hline$\tilde{Q}^{c \dagger} \bar{\Psi}^{c \dagger}$ & $P^{7} \bar{P}^{\dagger 2}$ & -122 \\
\hline$Q Q^{\dagger}$ & $\mathcal{H}^{2} P^{4}$ & -106 \\
\hline$Q \bar{\Psi}$ & $\mathcal{H}^{2} P^{4} \bar{P}^{\dagger}$ & -122 \\
\hline$Q \Psi^{\dagger}$ & $P^{6} \bar{P}^{\dagger 2}$ & -106 \\
\hline$Q^{\dagger} \Psi$ & $P^{8}$ & -106 \\
\hline Operator $\mathcal{O} \in K_{\mathrm{PQ}}$ & Coupling & $\log _{10} \Delta \theta$ \\
\hline$Q^{\dagger} \bar{\Psi}^{\dagger}$ & $P^{7} w_{0}$ & -116 \\
\hline$\overline{\bar{S}} c S^{c}$ & $\mathcal{H}^{2} P^{4}$ & -106 \\
\hline $\bar{S}^{c} \Psi^{c}$ & $\mathcal{H}^{2} P^{3} \bar{P}^{\dagger 2}$ & -122 \\
\hline $\bar{S}^{c} \bar{\Psi}^{c \dagger}$ & $P^{8} w_{0}$ & -132 \\
\hline$S^{c} \bar{\Psi}^{c}$ & $\mathcal{H}^{2} P^{3} \bar{P}^{\dagger}$ & -106 \\
\hline$S^{c} \Psi^{c \dagger}$ & $P^{6} \bar{P}^{\dagger 3}$ & -122 \\
\hline$X^{2}$ & $\mathcal{H}^{2} P^{4}$ & -106 \\
\hline$\Sigma^{2}$ & $P^{3} \bar{P}^{\dagger 3}$ & -74 \\
\hline$\Sigma \bar{\Sigma}$ & $\mathcal{H}^{2} P^{4}$ & -106 \\
\hline $\bar{\Sigma}^{2}$ & $P^{2} \bar{P}^{\dagger 2}$ & -42 \\
\hline$\Psi \bar{\Psi}$ & $P^{8} \bar{P}^{\dagger}$ & -122 \\
\hline$\Psi \Psi^{\dagger}$ & $\mathcal{H}^{2} P^{4}$ & -106 \\
\hline $\bar{\Psi} \bar{\Psi} \dagger$ & $\mathcal{H}^{2} P^{4}$ & -106 \\
\hline $\bar{\Psi} \dagger \Psi^{\dagger}$ & $P^{6} \bar{P}^{\dagger}$ & -90 \\
\hline$\Psi^{c} \bar{\Psi}^{c}$ & $P^{7}$ & -90 \\
\hline$\Psi^{c} \Psi^{c \dagger}$ & $\mathcal{H}^{2} P^{4}$ & -106 \\
\hline $\bar{\Psi}^{c} \bar{\Psi}^{c \dagger}$ & $\mathcal{H}^{2} P^{4}$ & -106 \\
\hline $\bar{\Psi} \bar{\Psi}^{c \dagger} \Psi^{c \dagger}$ & $P^{7} \bar{P}^{\dagger 2}$ & -122 \\
\hline $\mathcal{H} Q S^{c}$ & $\mathcal{H}^{2} P^{3} \bar{P}^{\dagger 2}$ & -126 \\
\hline$\hat{\mathcal{H}} \hat{S}^{c} \Psi$ & $P^{7} \bar{P}^{\dagger 2}$ & -126 \\
\hline $\mathcal{H} S^{c} \overline{\boldsymbol{\Psi}}^{\dagger}$ & $\mathcal{H}^{2} P^{3} \bar{P}^{\dagger}$ & -110 \\
\hline$\overline{\mathcal{H}} Q S^{c}$ & $P^{7} w_{0}$ & -120 \\
\hline$\overline{\mathcal{H}} S^{c} \Psi$ & $\mathcal{H}^{2} P^{3} \bar{P}^{\dagger 2}$ & -126 \\
\hline$\overline{\mathcal{H}} S^{c} \bar{\Psi}^{\dagger}$ & $P^{5} \bar{P}^{\dagger 3}$ & -110 \\
\hline$Q^{c} S^{c} \Sigma$ & $P^{6} \bar{P}^{\dagger 3}$ & -126 \\
\hline$Q^{c} S^{c} \bar{\Sigma}$ & $P^{3}$ & -30 \\
\hline$S^{c} \Sigma \Psi^{c}$ & $P^{6} \bar{P}^{\dagger 3}$ & -126 \\
\hline$S^{c} \Sigma \bar{\Psi}^{c \dagger}$ & $P^{8}$ & -110 \\
\hline$S^{c} \bar{\Sigma} \Psi^{c}$ & $P^{3}$ & -30 \\
\hline$S^{c} \bar{\Sigma} \bar{\Psi}^{c \dagger}$ & $\mathcal{H}^{2}$ & -46 \\
\hline $\mathcal{H} Q^{\dagger} \bar{S}^{c}$ & $\mathcal{H}^{2} P^{4} \bar{P}^{\dagger}$ & -126 \\
\hline
\end{tabular}

(Table continued)
TABLE VIII. (Continued)

\begin{tabular}{lcc}
\hline \hline Operator $\mathcal{O} \in K_{\mathrm{PQ}}$ & Coupling & $\log _{10} \Delta \theta$ \\
\hline $\mathcal{H} \bar{S}^{c} \bar{\Psi}^{\dagger}$ & $\mathcal{H}^{2} P^{4} \bar{P}^{\dagger 2}$ & -142 \\
$\mathcal{H} \bar{S}^{c} \Psi^{\dagger}$ & $P^{6} \bar{P}^{\dagger 3}$ & -126 \\
$\overline{\mathcal{H}} Q^{\dagger} \bar{S}^{c}$ & $P^{6} \bar{P}^{\dagger 3}$ & -126 \\
$\overline{\mathcal{H}} \bar{S}^{c} \bar{\Psi}^{\dagger}$ & $P^{8} w_{0}$ & -136 \\
$\overline{\mathcal{H}} \bar{S}^{c} \Psi^{\dagger}$ & $P^{7}$ & -94 \\
$Q^{c \dagger} \bar{S}^{c} \Sigma$ & $\mathcal{H}^{2} P$ & -62 \\
$Q^{c \dagger} \bar{S}^{c} \bar{\Sigma}$ & $\mathcal{H}^{2} P^{3} \bar{P}^{\dagger 2}$ & -126 \\
$\bar{S}^{c} \Sigma \bar{\Psi}^{c}$ & $P^{4}$ & -46 \\
$\bar{S}^{c} \Sigma \Psi^{c \dagger}$ & $\mathcal{H}^{2} P$ & -62 \\
$\bar{S}^{c} \bar{\Sigma} \bar{\Psi}^{c}$ & $P^{6} \bar{P}^{\dagger 2}$ & -110 \\
$\bar{S}^{c} \bar{\Sigma} \Psi^{c \dagger}$ & $\mathcal{H}^{2} P^{3} \bar{P}^{\dagger 2}$ & -126 \\
\hline \hline
\end{tabular}

TABLE IX. Sizes of masses (left) and coupling constants for dimension-4 and -5 operators which can be relevant to proton decay (right) in the RPV model.

\begin{tabular}{|c|c|c|}
\hline Operator $\mathcal{O}$ & Mass $m_{\mathcal{O}}$ & $\log _{10} m_{\mathcal{O}}$ \\
\hline $\mathcal{H}^{2}$ & $P \bar{P}$ & 2 \\
\hline$\overline{\mathcal{H}} \mathcal{H}$ & $w_{0}$ & 5 \\
\hline$\overline{\mathcal{H}}^{2}$ & $P \bar{P}^{3} w_{0}$ & -27 \\
\hline$Q^{c} \bar{S}^{c}$ & $P \bar{P}^{2}$ & -6 \\
\hline$\hat{Q}^{c} \bar{\Psi}^{c}$ & $\bar{P}$ & 10 \\
\hline$\overline{\bar{S}}^{c} S^{c}$ & $w_{0}$ & 5 \\
\hline $\bar{S}^{c} \Psi^{c}$ & $P \bar{P}^{2}$ & -6 \\
\hline$S^{c} \bar{\Psi}^{c}$ & $P \bar{P}^{3}$ & -14 \\
\hline$X^{2}$ & $w_{0}$ & 5 \\
\hline$\Sigma^{2}$ & $\bar{P}^{6}$ & -30 \\
\hline$\Sigma \bar{\Sigma}$ & $w_{0}$ & 5 \\
\hline $\bar{\Sigma}^{2}$ & $P^{4} \bar{P}^{2}$ & -30 \\
\hline$Q \bar{\Psi}$ & $P^{2} \bar{P}^{3}$ & -22 \\
\hline$\widetilde{\Psi} \bar{\Psi}$ & $P$ & 10 \\
\hline$\Psi^{c} \bar{\Psi}^{c}$ & $\bar{P}$ & 10 \\
\hline $\bar{S}^{c} S^{c} X$ & 1 & 16 \\
\hline$S^{c} S^{c} \Sigma$ & 1 & 16 \\
\hline $\bar{S}^{c} \bar{S}^{c} \bar{\Sigma}$ & 1 & 16 \\
\hline $\mathcal{H} Q S^{c}$ & $P \bar{P}^{2}$ & -8 \\
\hline $\mathcal{H} S^{c} \Psi$ & $P^{2} \bar{P}^{3} w_{0}$ & -37 \\
\hline$\overline{\mathcal{H}} Q S^{c}$ & $\bar{P} w_{0}$ & -5 \\
\hline$\overline{\mathcal{H}} S^{c} \Psi$ & $P \bar{P}^{2}$ & -8 \\
\hline$Q^{c} S^{c} \Sigma$ & $P \bar{P}^{2} w_{0}$ & -21 \\
\hline$Q^{c} S^{c} \bar{\Sigma}$ & $P^{3} w_{0}$ & -21 \\
\hline$S^{c} \Sigma \Psi^{c}$ & $P \bar{P}^{2} w_{0}$ & -21 \\
\hline$S^{c} \bar{\Sigma} \Psi^{c}$ & $P^{3} w_{0}$ & -21 \\
\hline $\mathcal{H} \bar{S}^{c} \bar{\Psi}$ & $P^{2} \bar{P}^{2}$ & -16 \\
\hline$\overline{\mathcal{H}} \bar{S}^{c} \bar{\Psi}$ & $P \bar{P} w_{0}$ & -13 \\
\hline $\bar{S}^{c} \sum \bar{\Psi}^{c}$ & $P^{4} w_{0}$ & -29 \\
\hline $\bar{S}^{c} \bar{\Sigma} \bar{\Psi}^{c}$ & $P \bar{P}^{3} w_{0}$ & -29 \\
\hline Operator $\mathcal{O}$ & $\lambda_{\mathcal{O}}$ or $\kappa_{\mathcal{O}}$ & $\log _{10} \lambda_{\mathcal{O}}, \kappa_{\mathcal{O}}$ \\
\hline$Q^{2} Q^{c} S^{c}$ & $\bar{P}$ & -10 \\
\hline$Q^{c 3} S^{c}$ & $P^{3} w_{0}$ & -39 \\
\hline
\end{tabular}

(Table continued) 
TABLE IX. (Continued)

\begin{tabular}{lcc}
\hline \hline Operator $\mathcal{O}$ & $\lambda_{\mathcal{O}}$ or $\kappa_{\mathcal{O}}$ & $\log _{10} \lambda_{\mathcal{O}}, \kappa_{\mathcal{O}}$ \\
\hline$Q^{4}$ & $\mathcal{H}^{2} w_{0}$ & -63 \\
$Q^{c 4}$ & $P^{4} \bar{P}^{2}$ & -66 \\
$Q \Sigma \Psi$ & $\bar{P}^{6}$ & -48 \\
$Q \bar{\Sigma} \Psi$ & $w_{0}$ & -13 \\
$Q^{3} \Psi$ & $P^{4} w_{0}$ & -63 \\
$Q Q^{c 2} \Psi$ & $w_{0}$ & -31 \\
$Q Q^{c} S^{c} \Psi$ & $P \bar{P}^{2} w_{0}$ & -39 \\
$Q Q^{c} \bar{S}^{c} \Sigma \Psi$ & $\mathcal{H}^{2} P w_{0}$ & -73 \\
$Q Q^{c} \bar{S}^{c} \bar{\Psi}$ & $P \bar{P}$ & -18 \\
$Q Q^{c} S^{c} \bar{\Sigma} \bar{\Psi}$ & $\mathcal{H}^{2}$ & -52 \\
$Q^{c} \Sigma \Psi^{c}$ & $w_{0}$ & -13 \\
$Q^{c} \bar{\Sigma} \Psi^{c}$ & $P^{4} \bar{P}^{2}$ & -48 \\
$Q^{2} Q^{c} \Psi$ & $P \bar{P}^{3} w_{0}$ & -63 \\
$Q^{2} S^{c} \Psi^{c}$ & $\bar{P}$ & -10 \\
$Q^{c 3} \Psi^{c}$ & $P^{4} \bar{P}^{2}$ & -66 \\
\hline
\end{tabular}

(Table continued)

larger than $10^{-20}$ only for the tree-level PQ breaking effects. The exotic mass terms, masses not included in $W_{\mathrm{PS}}, W_{\mathrm{PQ}}$ nor RPV superpotential in Eq. (61), are less than $10^{-13} \mathrm{GeV}$ which are negligible. The cubic and quartic operators for RPV and proton decay are suppressed as $\lambda_{\mathcal{O}} \lesssim 10^{-18}, \kappa_{\mathcal{O}}<10^{-31} \mathrm{GeV}^{-1}$ except the couplings in
TABLE IX. (Continued)

\begin{tabular}{lcc}
\hline \hline Operator $\mathcal{O}$ & $\lambda_{\mathcal{O}}$ or $\kappa_{\mathcal{O}}$ & $\log _{10} \lambda_{\mathcal{O}}, \kappa_{\mathcal{O}}$ \\
\hline$Q^{c 2} S^{c} \Psi^{c}$ & $P^{3} w_{0}$ & -39 \\
$Q^{2} \bar{S}^{c} \Sigma \Psi^{c}$ & $\bar{P}^{7}$ & -76 \\
$Q^{c 2} \bar{S}^{c} \Sigma \Psi^{c}$ & $P \bar{P}^{2}$ & -44 \\
$Q^{2} \bar{S}^{c} \bar{\Psi}^{c}$ & $\mathcal{H}^{2} w_{0}$ & -47 \\
$Q^{c 2} \bar{S}^{c} \bar{\Psi}^{c}$ & $P \bar{P}^{3} w_{0}$ & -47 \\
$Q^{2} S^{c} \bar{\Sigma} \bar{\Psi}^{c}$ & $\bar{P}^{2} w_{0}$ & -49 \\
$Q^{c 2} S^{c} \bar{\Sigma} \bar{\Psi}^{c}$ & $P^{3} \bar{P}$ & -52 \\
$Q^{2} \Sigma$ & $P^{4} w_{0}$ & -45 \\
$Q^{c 2} \Sigma$ & $w_{0}$ & -13 \\
$Q^{2} Q^{c} \bar{S}^{c} \Sigma$ & $\bar{P}^{7}$ & -76 \\
$Q^{c 3} \bar{S}^{c} \Sigma$ & $P \bar{P}^{2}$ & -44 \\
$Q^{2} \bar{\Sigma}$ & $P \bar{P}^{3} w_{0}$ & -45 \\
$Q^{c 2} \bar{\Sigma}$ & $P^{4} \bar{P}^{2}$ & -48 \\
$Q^{2} Q^{c} \bar{S}^{c} \bar{\Sigma}$ & $\bar{P}_{w_{0}}$ & -41 \\
$Q^{c 3} \bar{S}^{c} \bar{\Sigma}$ & $P^{3}$ & -44 \\
\hline \hline
\end{tabular}

Eq. (61). Most of the operators satisfy the sufficient conditions for proton stability as discussed in Sec. II E and the other operators shown in Eq. (61) will not induce too fast proton decay as discussed in Sec. III B. Therefore the proton will not be destabilized by these couplings.
[1] R. Peccei and H. R. Quinn, $C P$ Conservation in the Presence of Instantons, Phys. Rev. Lett. 38, 1440 (1977).

[2] R. Peccei and H. R. Quinn, Constraints imposed by $C P$ conservation in the presence of instantons, Phys. Rev. D 16, 1791 (1977).

[3] S. Weinberg, A New Light Boson?, Phys. Rev. Lett. 40, 223 (1978).

[4] F. Wilczek, Problem of Strong $P$ and $T$ Invariance in the Presence of Instantons, Phys. Rev. Lett. 40, 279 (1978).

[5] C. Baker et al., An Improved Experimental Limit on the Electric Dipole Moment of the Neutron, Phys. Rev. Lett. 97, 131801 (2006).

[6] J. M. Pendlebury et al., Revised experimental upper limit on the electric dipole moment of the neutron, Phys. Rev. D 92, 092003 (2015).

[7] B. Graner, Y. Chen, E. Lindahl, and B. Heckel, Reduced Limit on the Permanent Electric Dipole Moment of $\mathrm{Hg} 199$, Phys. Rev. Lett. 116, 161601 (2016); Erratum, Phys. Rev. Lett. 119, 119901 (2017).

[8] H. M. Georgi, L. J. Hall, and M. B. Wise, Grand unified models with an automatic Peccei-Quinn symmetry, Nucl. Phys. B192, 409 (1981).

[9] M. Dine and N. Seiberg, String theory and the strong $C P$ problem, Nucl. Phys. B273, 109 (1986).
[10] M. Kamionkowski and J. March-Russell, Planck scale physics and the Peccei-Quinn mechanism, Phys. Lett. B 282, 137 (1992).

[11] R. Holman, S. D. Hsu, T. W. Kephart, E. W. Kolb, R. Watkins, and L. M. Widrow, Solutions to the strong $C P$ problem in a world with gravity, Phys. Lett. B 282, 132 (1992).

[12] S. Ghigna, M. Lusignoli, and M. Roncadelli, Instability of the invisible axion, Phys. Lett. B 283, 278 (1992).

[13] L. Randall, Composite axion models and Planck scale physics, Phys. Lett. B 284, 77 (1992).

[14] K. Izawa, T. Watari, and T. Yanagida, Higher dimensional QCD without the strong $C P$ problem, Phys. Lett. B 534, 93 (2002).

[15] K.-w. Choi, A QCD Axion From Higher Dimensional Gauge Field, Phys. Rev. Lett. 92, 101602 (2004).

[16] K. Harigaya, M. Ibe, K. Schmitz, and T. T. Yanagida, PecceiQuinn symmetry from a gauged discrete R symmetry, Phys. Rev. D 88, 075022 (2013).

[17] B. Lillard and T. M. Tait, A high quality composite axion, J. High Energy Phys. 11 (2018) 199.

[18] A. Hook, S. Kumar, Z. Liu, and R. Sundrum, The High Quality QCD Axion and the LHC, Phys. Rev. Lett. 124, 221801 (2020). 
[19] M. Ardu, L. Di Luzio, G. Landini, A. Strumia, D. Teresi, and J.-W. Wang, Axion quality from the (anti)symmetric of SU(N), J. High Energy Phys. 11 (2020) 090.

[20] W. Yin, Scale and quality of Peccei-Quinn symmetry and weak gravity conjectures, J. High Energy Phys. 10 (2020) 032 .

[21] L. Di Luzio, Accidental SO(10) axion from gauged flavour, J. High Energy Phys. 11 (2020) 074.

[22] J. C. Pati and A. Salam, Lepton number as the fourth color, Phys. Rev. D 10, 275 (1974); Errtaum, Phys. Rev. D 11, 703 (1975).

[23] T. Kobayashi, S. Raby, and R.-J. Zhang, Constructing 5-D orbifold grand unified theories from heterotic strings, Phys. Lett. B 593, 262 (2004).

[24] T. Kobayashi, S. Raby, and R.-J. Zhang, Searching for realistic 4d string models with a Pati-Salam symmetry: Orbifold grand unified theories from heterotic string compactification on a Z(6) orbifold, Nucl. Phys. B704, 3 (2005).

[25] Z. Poh and S. Raby, Yukawa Unification in an SO(10) SUSY GUT: SUSY on the edge, Phys. Rev. D 92, 015017 (2015).

[26] Z. Poh, S. Raby, and Z.-z. Wang, Pati-Salam SUSY GUT with Yukawa unification, Phys. Rev. D 95, 115025 (2017).

[27] H. M. Lee, S. Raby, M. Ratz, G. G. Ross, R. Schieren, K. Schmidt-Hoberg, and P. K. Vaudrevange, A unique $\mathbb{Z}_{4}^{R}$ symmetry for the MSSM, Phys. Lett. B 694, 491 (2011).

[28] H. M. Lee, S. Raby, M. Ratz, G. G. Ross, R. Schieren, K. Schmidt-Hoberg, and P. K. Vaudrevange, Discrete R symmetries for the MSSM and its singlet extensions, Nucl. Phys. B850, 1 (2011).

[29] T. R. Slatyer and C.-L. Wu, General constraints on dark matter decay from the cosmic microwave background, Phys. Rev. D 95, 023010 (2017).

[30] M. Kawasaki and T. Moroi, Gravitino production in the inflationary universe and the effects on big bang nucleosynthesis, Prog. Theor. Phys. 93, 879 (1995).

[31] M. Kawasaki, K. Kohri, and T. Moroi, Big-Bang nucleosynthesis and hadronic decay of long-lived massive particles, Phys. Rev. D 71, 083502 (2005).

[32] M. Kawasaki, K. Kohri, T. Moroi, and A. Yotsuyanagi, Big-bang nucleosynthesis and gravitino, Phys. Rev. D 78, 065011 (2008).

[33] M. Kawasaki, K. Kohri, T. Moroi, and Y. Takaesu, Revisiting big-bang nucleosynthesis constraints on long-lived decaying particles, Phys. Rev. D 97, 023502 (2018).

[34] C. Froggatt and H. B. Nielsen, Hierarchy of quark masses, Cabibbo Angles and CP violation, Nucl. Phys. B147, 277 (1979).

[35] B. C. Bryant and S. Raby, Pati-Salam version of subcritical hybrid inflation, Phys. Rev. D 93, 095003 (2016).

[36] G. Lazarides, M. U. Rehman, Q. Shafi, and F. K. Vardag, Shifted $\mu$-hybrid inflation, gravitino dark matter, and observable gravity waves, arXiv:2007.01474.

[37] J. Casas and C. Munoz, A natural solution to the mu problem, Phys. Lett. B 306, 288 (1993).

[38] J. E. Kim, Weak Interaction Singlet and Strong CP iznvariance, Phys. Rev. Lett. 43, 103 (1979).

[39] M. A. Shifman, A. Vainshtein, and V. I. Zakharov, Can confinement ensure natural $C P$ invariance of strong interactions?, Nucl. Phys. B166, 493 (1980).
[40] P. Moxhay and K. Yamamoto, Peccei-Quinn symmetry breaking by radiative corrections in supergravity, Phys. Lett. 151B, 363 (1985).

[41] S. R. Coleman and E. J. Weinberg, Radiative corrections as the origin of spontaneous symmetry breaking, Phys. Rev. D 7, 1888 (1973).

[42] J. E. Kim and H. P. Nilles, The mu problem and the strong CP problem, Phys. Lett. 138B, 150 (1984).

[43] L. E. Ibanez and G. G. Ross, Discrete gauge symmetries and the origin of baryon and lepton number conservation in supersymmetric versions of the standard model, Nucl. Phys. B368, 3 (1992).

[44] L. E. Ibanez and G. G. Ross, Discrete gauge symmetry anomalies, Phys. Lett. B 260, 291 (1991).

[45] L. E. Ibanez, More about discrete gauge anomalies, Nucl. Phys. B398, 301 (1993).

[46] H. K. Dreiner, C. Luhn, and M. Thormeier, What is the discrete gauge symmetry of the MSSM?, Phys. Rev. D 73, 075007 (2006).

[47] T. Araki, Anomaly of discrete symmetries and gauge coupling unification, Prog. Theor. Phys. 117, 1119 (2007).

[48] T. Araki, T. Kobayashi, J. Kubo, S. Ramos-Sanchez, M. Ratz, and P. K. S. Vaudrevange, (Non-)Abelian discrete anomalies, Nucl. Phys. B805, 124 (2008).

[49] M. B. Green and J. H. Schwarz, Anomaly cancellation in supersymmetric $D=10$ gauge theory and superstring theory, Phys. Lett. 149B, 117 (1984).

[50] V. Berezinsky, A. S. Joshipura, and J. Valle, Gravitational violation of R-parity and its cosmological signatures, Phys. Rev. D 57, 147 (1998).

[51] R. Barbier et al., R-parity violating supersymmetry, Phys. Rep. 420, 1 (2005).

[52] P. Sikivie, Of Axions, Domain Walls and the Early Universe, Phys. Rev. Lett. 48, 1156 (1982).

[53] M. Ibe, S. Kobayashi, M. Suzuki, and T. T. Yanagida, Dynamical solution to the axion domain wall problem, Phys. Rev. D 101, 035029 (2020).

[54] M. Cirelli, N. Fornengo, and A. Strumia, Minimal dark matter, Nucl. Phys. B753, 178 (2006).

[55] M. Cirelli, A. Strumia, and M. Tamburini, Cosmology and astrophysics of minimal dark matter, Nucl. Phys. B787, 152 (2007).

[56] M. Endo, K. Hamaguchi, and F. Takahashi, Moduli-Induced Gravitino Problem, Phys. Rev. Lett. 96, 211301 (2006).

[57] K.-Y. Choi, L. Covi, J. E. Kim, and L. Roszkowski, Axino cold dark matter revisited, J. High Energy Phys. 04 (2012) 106.

[58] J. E. Kim and M.-S. Seo, Mixing of axino and goldstino, and axino mass, Nucl. Phys. B864, 296 (2012).

[59] K.-Y. Choi, J. E. Kim, and L. Roszkowski, Review of axino dark matter, J. Korean Phys. Soc. 63, 1685 (2013).

[60] H.-B. Kim and J. E. Kim, Late decaying axino as CDM and its lifetime bound, Phys. Lett. B 527, 18 (2002).

[61] D. Hooper and L.-T. Wang, Possible evidence for axino dark matter in the galactic bulge, Phys. Rev. D 70, 063506 (2004).

[62] E. J. Chun and H. B. Kim, Axino light dark matter and neutrino masses with R-parity violation, J. High Energy Phys. 10 (2006) 082. 
[63] M. Endo, K. Hamaguchi, S. P. Liew, K. Mukaida, and K. Nakayama, Axino dark matter with R-parity violation and $130 \mathrm{GeV}$ gamma-ray line, Phys. Lett. B 721, 111 (2013).
[64] R. T. Co and K. Harigaya, Axiogenesis, Phys. Rev. Lett. 124, 111602 (2020).

[65] R. T. Co, N. Fernandez, A. Ghalsasi, L. J. Hall, and K. Harigaya, Lepto-axiogenesis, arXiv:2006.05687. 